\title{
Smarandache Curves of Mannheim Curve Couple According to Frenet Frame
}

\author{
Süleyman Şenyurt* and Abdussamet Çalışkan
}

(Communicated by Kazım İLARSLAN)

\begin{abstract}
In this paper, when the Frenet vectors of the partner curve of Mannheim curve are taken as the position vectors, the curvature and the torsion of Smarandache curves are calculated. These values are expressed depending upon the Mannheim curve. Besides, we illustrate example of our main results.

Keywords: Mannheim curve, Mannheim partner curve, Smarandache Curves, Frenet invariants

AMS Subject Classification (2010): $53 A 04$

*Corresponding author
\end{abstract}

\section{Introduction}

A regular curve in Minkowski space-time, whose position vector is composed by Frenet frame vectors on another regular curve, is called a Smarandache curve [10]. Special Smarandache curves have been studied by some authors .

Melih Turgut and Süha Y1lmaz studied a special case of such curves and called it Smarandache $T B_{2}$ curves in the space $E_{1}^{4}$ [10]. Ahmad T.Ali studied some special Smarandache curves in the Euclidean space. He studied Frenet-Serret invariants of a special case [1]. Muhammed Çetin, Yılmaz Tunçer and Kemal Karacan investigated special Smarandache curves according to Bishop frame in Euclidean 3-Space and they gave some differential goematric properties of Smarandache curves, also they found the centers of the osculating spheres and curvature spheres of Smarandache curves [5]. Şenyurt and Çalışkan investigated special Smarandache curves in terms of Sabban frame of spherical indicatrix curves and they gave some characterization of Smarandache curves.[4] Özcan Bektaş and Salim Yüce studied some special Smarandache curves according to Darboux Frame in $E^{3}$ [2]. Nurten Bayrak, Özcan Bektaş and Salim Yüce studied some special Smarandache curves in $E_{1}^{3}$ [3].

In this paper, special Smarandache curves belonging to $\alpha^{*}$ Mannheim partner curve such as $T^{*} N^{*}, N^{*} B^{*}, T^{*} B^{*}$ and $T^{*} N^{*} B^{*}$ drawn by Frenet frame are defined and some related results are given.

\section{Preliminaries}

The Euclidean 3-space $E^{3}$ be inner product given by

$$
\langle,\rangle=x_{1}^{2}+x_{2}^{3}+x_{3}^{2}
$$

Received : 11-November-2016, Accepted : 17-April-2017 
where $\left(x_{1}, x_{2}, x_{3}\right) \in E^{3}$. Let $\alpha: I \rightarrow E^{3}$ be a unit speed curve denote by $\{T, N, B\}$ the moving Frenet frame. For an arbitrary curve $\alpha \in E^{3}$, with first and second curvature, $\kappa$ and $\tau$ respectively, the Frenet formulae is given by [6], [9]

$$
\left\{\begin{array}{l}
T^{\prime}=\kappa N \\
N^{\prime}=-\kappa T+\tau B \\
B^{\prime}=-\tau N .
\end{array}\right.
$$

Let $\alpha: I \rightarrow \mathbb{E}^{3}$ and $\alpha^{*}: I \rightarrow \mathbb{E}^{3}$ be the $C^{2}$ - class differentiable unit speed two curves and let $\{T(s), N(s), B(s)\}$ and $\left\{T^{*}(s), N^{*}(s), B^{*}(s)\right\}$ be the Frenet frames of the curves $\alpha$ and $\alpha^{*}$, respectively. If the principal normal vector $N$ of the curve $\alpha$ is linearly dependent on the binormal vector $B$ of the curve $\alpha^{*}$, then $(\alpha)$ is called a Mannheim curve and $\left(\alpha^{*}\right)$ a Mannheim partner curve of $(\alpha)$. The pair $\left(\alpha, \alpha^{*}\right)$ is said to be Mannheim pair [7], [8]. The relations between the Frenet frames $\{T(s), N(s), B(s)\}$ and $\left\{T^{*}(s), N^{*}(s), B^{*}(s)\right\}$ are as follows:

$$
\begin{gathered}
\left\{\begin{array}{l}
T^{*}=\cos \theta T-\sin \theta B \\
N^{*}=\sin \theta T+\cos \theta B \\
B^{*}=N
\end{array}\right. \\
\left\{\begin{array}{l}
\cos \theta=\frac{d s *}{d s} \\
\sin \theta=\lambda \tau^{*} \frac{d s *}{d s}
\end{array}\right.
\end{gathered}
$$

where $\angle\left(T, T^{*}\right)=\theta$, [8].

Theorem 2.1. The distance between corresponding points of the Mannheim partner curves in $\mathbb{E}^{3}$ is constant, [7].

Theorem 2.2. Let $\left(\alpha, \alpha^{*}\right)$ be a Mannheim pair curves in $\mathbb{E}^{3}$. For the curvatures and the torsions of the Mannheim curve pair $\left(\alpha, \alpha^{*}\right)$ we have,

$$
\left\{\begin{array}{l}
\kappa=\tau^{*} \sin \theta \frac{d s *}{d s} \\
\tau=-\tau^{*} \cos \theta \frac{d s *}{d s}
\end{array}\right.
$$

and

$$
\left\{\begin{array}{l}
\kappa^{*}=\frac{d \theta}{d s *}=\theta^{\prime} \frac{\kappa}{\lambda \tau \sqrt{\kappa^{2}+\tau^{2}}} \\
\tau^{*}=(\kappa \sin \theta-\tau \cos \theta) \frac{d s *}{d s}
\end{array}\right.
$$

Theorem 2.3. Let $\left(\alpha, \alpha^{*}\right)$ be a Mannheim pair curves in $\mathbb{E}^{3}$. For the torsions $\tau^{*}$ of the Mannheim partner curve $\alpha^{*}$ we have

$$
\tau^{*}=\frac{\kappa}{\lambda \tau}
$$

\section{Smarandache Curves of Mannheim Curve Couple According to Frenet Frame}

Let $\left(\alpha, \alpha^{*}\right)$ be a Mannheim pair curves in $E^{3}$ and $\left\{T^{*} N^{*} B^{*}\right\}$ be the Frenet frame of the Mannheim partner curve $\alpha^{*}$ at $\alpha^{*}(s)$. In this case, $T^{*} N^{*}$ - Smarandache curve can be defined by

$$
\beta_{1}(s)=\frac{1}{\sqrt{2}}\left(T^{*}+N^{*}\right) .
$$

Solving the above equation by substitution of $T^{*}$ and $N^{*}$ from (2.2), we obtain

$$
\beta_{1}(s)=\frac{(\cos \theta+\sin \theta) T+(\cos \theta-\sin \theta) B}{\sqrt{2}} .
$$

The derivative of this equation with respect to $s$ is as follows, 


$$
\beta_{1}^{\prime}=T_{\beta_{1}} \frac{d s_{\beta_{1}}}{d s}=\frac{\theta^{\prime} \kappa(\sin \theta-\cos \theta) T+\kappa \sqrt{\kappa^{2}+\tau^{2}} N+\kappa\left(\theta^{\prime} \sin \theta+\theta^{\prime} \cos \theta\right) B}{\sqrt{2 \tau^{2} \lambda^{2}\|W\|}}
$$

and by substitution, we get

$$
T_{\beta_{1}}(s)=\frac{\theta^{\prime} \kappa(\sin \theta-\cos \theta) T+\kappa \sqrt{\kappa^{2}+\tau^{2}} N+\kappa\left(\theta^{\prime} \sin \theta+\theta^{\prime} \cos \theta\right) B}{\sqrt{\kappa^{2}\left(2{\theta^{\prime}}^{2}+\kappa^{2}+\tau^{2}\right)}}
$$

where

$$
\frac{d s_{\beta_{1}}}{d s}=\sqrt{\frac{\kappa^{2}\left(2{\theta^{\prime}}^{2}+\kappa^{2}+\tau^{2}\right)}{2 \tau^{2} \lambda^{2}\|W\|}} .
$$

In order to determine the first curvature and the principal normal of the curve $\beta_{1}(s)$, we formalize

$$
T_{\beta_{1}}^{\prime}(s)=\sqrt{2} \frac{\lambda^{4} \tau^{4}\left(\kappa^{2}+\tau^{2}\right)^{2}}{\kappa^{4}\left(2 \theta^{\prime 2}+\kappa^{2}+\tau^{2}\right)^{2}}\left[\left(\overline{\ell_{1}} \cos \theta+\overline{\ell_{2}} \sin \theta\right) T+\overline{\ell_{3}} N+\left(\overline{\ell_{2}} \cos \theta-\overline{\ell_{1}} \sin \theta\right) B\right],
$$

where

$$
\left\{\begin{aligned}
\overline{\ell_{1}}= & -\frac{\theta^{\prime 2} \kappa^{4}\left(2 \theta^{\prime 2}+\kappa^{2}+\tau^{2}\right)}{\lambda^{4} \tau^{4}\left(\kappa^{2}+\tau^{2}\right)^{2}}-\frac{\kappa^{3}\left(\theta^{\prime \prime} \kappa^{2}+\theta^{\prime \prime} \tau^{2}-\theta^{\prime} \kappa \kappa^{\prime}-\theta^{\prime} \tau \tau^{\prime}\right)}{\lambda^{3} \tau^{3}\left(\kappa^{2}+\tau^{2}\right)^{\frac{3}{2}}} \\
\overline{\ell_{2}}= & -\frac{\kappa^{3}\left(\kappa^{3} \sqrt{\kappa^{2}+\tau^{2}}+\kappa \tau^{2} \sqrt{\kappa^{2}+\tau^{2}}-\theta^{\prime \prime} \lambda \tau \kappa^{2}-\theta^{\prime \prime} \lambda \tau^{3}+\theta^{\prime} \lambda \tau \kappa \kappa^{\prime}+\theta^{\prime} \lambda \tau^{2} \tau^{\prime}\right)}{\lambda^{4} \tau^{4}\left(\kappa^{2}+\tau^{2}\right)^{\frac{3}{2}}} \\
& -\frac{\theta^{\prime 2} \kappa^{4}\left(2 \theta^{\prime 2}+3 \kappa^{2}+3 \tau^{2}\right)}{\lambda^{4} \tau^{4}\left(\kappa^{2}+\tau^{2}\right)^{2}} \\
\overline{\ell_{3}}= & \frac{\theta^{\prime} \kappa^{4}\left(2 \theta^{\prime 2}+\kappa^{2}+\tau^{2}\right)}{\lambda^{4} \tau^{4}\left(\kappa^{2}+\tau^{2}\right)^{\frac{3}{2}}}-\frac{2 \theta^{\prime} \kappa^{3}\left(\theta^{\prime \prime} \kappa^{2}+\theta^{\prime \prime} \tau^{2}-\theta^{\prime} \kappa \kappa^{\prime}-\theta^{\prime} \tau \tau^{\prime}\right)}{\lambda^{3} \tau^{3}\left(\kappa^{2}+\tau^{2}\right)^{2}} .
\end{aligned}\right.
$$

The first curvature is

$$
\begin{aligned}
\kappa_{\beta_{1}} & =\left\|T_{\beta_{1}}^{\prime}\right\| \\
\kappa_{\beta_{1}} & =\sqrt{2} \frac{\lambda^{4} \tau^{4}\left(\kappa^{2}+\tau^{2}\right)^{2}}{\kappa^{4}\left(2 \theta^{\prime 2}+\kappa^{2}+\tau^{2}\right)^{2}} \sqrt{{\overline{\ell_{1}}}^{2}+{\overline{\ell_{2}}}^{2}+{\overline{\ell_{3}}}^{2}} .
\end{aligned}
$$

The principal normal vector field and the binormal vector field are respectively given by

$$
\begin{aligned}
& N_{\beta_{1}}=\frac{\left(\overline{\ell_{1}} \cos \theta+\overline{\ell_{2}} \sin \theta\right) T+\overline{\ell_{3}} N+\left(\overline{\ell_{2}} \cos \theta-\overline{\ell_{1}} \sin \theta\right) B}{\sqrt{{\overline{\ell_{1}}}^{2}+{\overline{\ell_{2}}}^{2}+{\overline{\ell_{3}}}^{2}}}, \\
& B_{\beta_{1}}=\frac{\left(\overline{\ell_{2}} \cos \theta-\overline{\ell_{1}} \sin \theta\right) \kappa\left(\|W\|+\theta^{\prime} \sin \theta\right)-\overline{\ell_{3}} \kappa \theta^{\prime} \cos \theta}{\sqrt{\kappa^{2}\left(\kappa^{2}+\tau^{2}+2{\theta^{\prime}}^{2}\right)\left({\overline{\ell_{1}}}^{2}+{\overline{\ell_{2}}}^{2}+{\overline{\ell_{3}}}^{2}\right)}} \mathbf{T} \\
& +\frac{\left(\overline{\ell_{1}} \cos \theta+\overline{\ell_{2}} \sin \theta\right) \kappa \theta^{\prime} \cos \theta-\left(\overline{\ell_{2}} \cos \theta-\overline{\ell_{1}} \sin \theta\right) \theta^{\prime} \kappa(\sin \theta-\cos \theta)}{\sqrt{\kappa^{2}\left(\kappa^{2}+\tau^{2}+2{\theta^{\prime}}^{2}\right)\left({\overline{\ell_{1}}}^{2}+{\overline{\ell_{2}}}^{2}+{\overline{\ell_{3}}}^{2}\right)}} \mathbf{N} \\
& +\frac{\overline{\ell_{3}} \theta^{\prime} \kappa(\sin \theta-\cos \theta)-\left(\overline{\ell_{1}} \cos \theta+\overline{\ell_{2}} \sin \theta\right) \kappa\left(\|W\|+\theta^{\prime} \sin \theta\right)}{\sqrt{\kappa^{2}\left(\kappa^{2}+\tau^{2}+2{\theta^{\prime}}^{2}\right)\left({\overline{\ell_{1}}}^{2}+{\overline{\ell_{2}}}^{2}+{\overline{\ell_{3}}}^{2}\right)}} \mathbf{B} .
\end{aligned}
$$


In order to calculate the torsion of the curve $\beta_{1}$, we differentiate

$$
\begin{aligned}
\beta_{1}^{\prime \prime}= & \frac{1}{\sqrt{2} \lambda^{3} \tau^{2}\|W\|^{2}}\left[\left(-\kappa^{4}\|W\| \sin \theta-\theta^{\prime 2} \kappa^{2}\|W\| \cos \theta-\theta^{\prime 2} \kappa^{2}\|W\| \sin \theta\right.\right. \\
& -\kappa^{2} \tau^{2}\|W\| \sin \theta+2 \kappa \tau^{2} \theta^{\prime} \lambda \tau^{\prime} \cos \theta-2 \kappa \tau^{2} \theta^{\prime} \lambda \tau^{\prime} \sin \theta-\kappa \tau^{3} \theta^{\prime} \lambda^{\prime} \sin \theta-\kappa \tau^{3} \lambda \theta^{\prime \prime} \cos \theta \\
& +\kappa \tau^{3} \theta^{\prime} \lambda^{\prime} \cos \theta+\tau^{3} \theta^{\prime} \lambda \kappa^{\prime} \sin \theta-\tau^{3} \theta^{\prime} \lambda \kappa^{\prime} \cos \theta-\kappa^{3} \theta^{\prime} \lambda \tau^{\prime} \sin \theta+\kappa \tau^{3} \lambda \theta^{\prime \prime} \sin \theta \\
& \left.-\kappa^{3} \tau \lambda \theta^{\prime \prime} \cos \theta-\kappa^{3} \tau \theta^{\prime} \lambda^{\prime} \sin \theta+\kappa^{3} \tau \theta^{\prime} \lambda^{\prime} \cos \theta+\kappa^{3} \theta^{\prime} \lambda \tau^{\prime} \cos \theta+\kappa^{3} \tau \lambda \theta^{\prime \prime} \sin \theta\right) \mathbf{T}+ \\
& \left(\kappa^{2} \tau^{2} \theta^{\prime}+\kappa^{4} \theta^{\prime}-\|W\| \kappa \tau^{3} \lambda^{\prime}+\|W\| \tau^{3} \lambda \kappa^{\prime}-\|W\| \kappa^{3} \tau \lambda^{\prime}-\|W\| \kappa^{3} \tau^{\prime}+\|W\| \kappa^{2} \tau \lambda \kappa^{\prime}\right. \\
& \left.-\|W\| \kappa \tau^{2} \tau^{\prime}\right) \mathbf{N}+\left(-\kappa^{4}\|W\| \cos \theta-\theta^{\prime 2} \kappa^{2}\|W\| \cos \theta+\theta^{\prime 2} \kappa^{2}\|W\| \sin \theta\right. \\
& -\kappa^{2} \tau^{2}\|W\| \cos \theta-2 \kappa \tau^{2} \theta^{\prime} \lambda \tau^{\prime} \sin \theta-2 \kappa \tau^{2} \theta^{\prime} \lambda \tau^{\prime} \cos \theta-\kappa \tau^{3} \theta^{\prime} \lambda^{\prime} \sin \theta+\kappa \tau^{3} \lambda \theta^{\prime \prime} \sin \theta \\
& +\kappa \tau^{3} \lambda \theta^{\prime \prime} \cos \theta+\tau^{3} \theta^{\prime} \lambda \kappa^{\prime} \sin \theta+\tau^{3} \theta^{\prime} \lambda \kappa^{\prime} \cos \theta-\kappa^{3} \tau \theta^{\prime} \lambda^{\prime} \cos \theta-\kappa^{3} \theta^{\prime} \lambda \tau^{\prime} \sin \theta \\
& \left.\left.+\kappa^{3} \tau \lambda \theta^{\prime \prime} \cos \theta-\kappa^{3} \theta^{\prime} \lambda \tau^{\prime} \cos \theta-\kappa^{3} \tau \theta^{\prime} \lambda^{\prime} \sin \theta+\kappa^{3} \tau \lambda \theta^{\prime \prime} \sin \theta-\kappa \tau^{3} \theta^{\prime} \lambda^{\prime} \cos \theta\right) \mathbf{B}\right] .
\end{aligned}
$$

and thus

$$
\beta_{1}^{\prime \prime \prime}=\frac{\left(\overline{\jmath_{1}} \cos \theta+\overline{\jmath_{2}} \sin \theta\right) T+\overline{\jmath_{3}} N+\left(-\overline{\jmath_{1}} \sin \theta+\overline{\jmath_{2}} \cos \theta\right) B}{\sqrt{2}}
$$

where

$$
\begin{aligned}
\overline{\jmath_{1}}= & \frac{2 \kappa^{5} \tau^{2} \theta^{\prime} \kappa^{3} \tau^{4} \theta^{\prime}+\kappa^{3} \tau^{2} \theta^{\prime 3}-3 \kappa^{4} \tau \theta^{\prime} \lambda \theta^{\prime \prime} \sqrt{\kappa^{2}+\tau^{2}}-3 \kappa^{2} \tau^{3} \theta^{\prime} \lambda \theta^{\prime \prime} \sqrt{\kappa^{2}+\tau^{2}}}{\lambda^{3} \tau^{3}\|W\|^{5}} \\
& -\frac{3 \kappa \tau^{3} \theta^{\prime 2} \lambda \kappa^{\prime} \sqrt{\kappa^{2}+\tau^{2}}-2 \kappa^{2} \tau^{4} \theta^{\prime} \lambda \kappa^{\prime} \lambda^{\prime}+6 \kappa^{3} \tau^{3} \theta^{\prime} \lambda \lambda^{\prime} \tau^{\prime}+4 \kappa \tau^{5} \theta^{\prime} \lambda \lambda^{\prime} \tau^{\prime}}{\lambda^{3} \tau^{3}\|W\|^{5}} \\
& -\frac{2 \kappa^{5} \tau \theta^{\prime} \lambda \lambda^{\prime} \tau^{\prime}-6 \theta^{\prime 2} \kappa^{2} \sqrt{\kappa^{2}+\tau^{2}} \tau^{2} \lambda \tau^{\prime}+2 \kappa^{2} \tau^{3} \theta^{\prime} \lambda^{2} \kappa^{\prime} \tau^{\prime}-3 \kappa^{3} \tau^{3} \theta^{\prime} \lambda^{2} \tau^{\prime \prime}}{\lambda^{3} \tau^{3}\|W\|^{5}} \\
& +\frac{2 \kappa \tau^{5} \theta^{\prime} \lambda^{2} \tau^{\prime \prime}-\kappa^{2} \tau^{4} \theta^{\prime} \lambda^{2} \kappa^{\prime}-2 \kappa^{2} \tau^{4} \lambda^{2} \kappa^{\prime} \theta^{\prime}+2 \kappa^{5} \tau^{2} \lambda \lambda^{\prime} \theta^{\prime \prime}+4 \kappa^{3} \tau^{4} \lambda \lambda^{\prime} \theta^{\prime \prime}}{\lambda^{3} \tau^{3}\|W\|^{5}} \\
& +\frac{2 \kappa \tau^{6} \lambda \lambda^{\prime} \theta^{\prime \prime}+2 \kappa^{5} \tau \lambda^{2} \tau^{\prime} \theta^{\prime \prime}+6 \kappa^{3} \tau^{3} \lambda^{2} \tau^{\prime \prime} \theta^{\prime \prime}-2 \kappa \tau^{6} \theta^{\prime} \lambda^{\prime 2}-2 \kappa^{5} \theta^{\prime} \lambda^{2} \tau^{\prime 2}}{\lambda^{3} \tau^{3}\|W\|^{5}} \\
& +\frac{4 \tau^{5} \lambda^{2} \tau^{\prime} \theta^{\prime \prime}+\kappa^{5} \tau^{2} \theta^{\prime} \lambda \lambda^{\prime \prime}+2 \kappa^{3} \tau^{4} \theta^{\prime} \lambda \lambda^{\prime \prime}+\kappa \tau^{6} \theta^{\prime} \lambda \lambda^{\prime \prime}+\kappa^{5} \tau \theta^{\prime} \lambda^{2} \tau^{\prime \prime}+\kappa^{5} \theta^{\prime 3}}{\lambda^{3} \tau^{3}\|W\|^{5}} \\
& +\frac{3 \kappa^{4} \theta^{\prime 2} \lambda \tau^{\prime} \sqrt{\kappa^{2}+\tau^{2}}+3 \kappa^{4} \tau \theta^{\prime 2} \lambda^{\prime} \sqrt{\kappa^{2}+\tau^{2}}+3 \kappa^{2} \tau^{3} \theta^{\prime 2} \lambda^{\prime} \sqrt{\kappa^{2}+\tau^{2}}}{\lambda^{3} \tau^{3}\|W\|^{5}} \\
& +\frac{2 \tau^{6} \theta^{\prime} \lambda \kappa^{\prime} \lambda^{\prime}+4 \tau^{5} \theta^{\prime} \lambda^{2} \kappa^{\prime} \tau^{\prime}-5 \kappa^{3} \tau^{2} \theta^{\prime} \lambda^{2} \tau^{\prime 2}-6 \kappa \tau^{4} \theta^{\prime} \lambda^{2} \tau^{\prime 2}+3 \kappa \tau^{4} \theta^{\prime} \lambda^{2} \kappa^{\prime 2}}{\lambda^{3} \tau^{3}\|W\|^{5}} \\
& +\frac{\kappa^{7} \theta^{\prime}-\tau^{6} \theta^{\prime} \lambda^{2} \kappa^{\prime \prime}-\kappa^{5} \tau^{2} \lambda^{2} \theta^{\prime \prime \prime}-2 \kappa^{3} \tau^{4} \lambda^{2} \theta^{\prime \prime \prime}-\kappa \tau^{6} \lambda^{2} \theta^{\prime \prime \prime}-2 \tau^{6} \lambda^{2} \kappa^{\prime} \theta^{\prime \prime}}{\lambda^{3} \tau^{3}\|W\|^{3} \|^{3} \tau^{4} \theta^{\prime} \lambda^{\prime 2}}
\end{aligned}
$$




$$
\begin{aligned}
& \int \overline{\jmath_{2}}=-\frac{3 \kappa^{5} \tau \lambda \kappa^{\prime}\|W\|+6 \kappa^{3} \tau^{3} \lambda \kappa^{\prime}\|W\|+3 \kappa \tau^{5} \lambda \kappa^{\prime}\|W\|-6 \kappa^{4} \tau^{2} \tau^{\prime} \lambda\|W\|}{\lambda^{3} \tau^{3}\|W\|^{5}} \\
& +\frac{3 \kappa^{2} \tau^{4} \tau^{\prime} \lambda\|W\|-\kappa \tau^{6} \theta^{\prime} \lambda \lambda^{\prime \prime}-\kappa^{5} \tau \theta^{\prime} \lambda^{2} \tau^{\prime \prime}+\kappa^{2} \tau^{4} \theta^{\prime} \lambda^{2} \kappa^{\prime \prime}+3 \kappa^{3} \tau^{3} \theta^{\prime} \lambda^{2} \tau^{\prime \prime}}{\lambda^{3} \tau^{3}\|W\|^{5}} \\
& -\frac{2 \kappa^{2} \tau^{4} \lambda^{2} \kappa^{\prime} \theta^{\prime \prime}-2 \kappa \tau^{5} \theta^{\prime} \lambda^{2} \tau^{\prime \prime}-6 \kappa^{3} \tau^{3} \lambda^{2} \tau^{\prime} \theta^{\prime \prime}-6 \kappa \tau^{4} \theta^{\prime} \lambda^{2} \tau^{\prime 2}+2 \kappa^{2} \tau^{4} \theta^{\prime} \lambda \kappa^{\prime} \lambda^{\prime}}{\lambda^{3} \tau^{3}\|W\|^{5}} \\
& -\frac{4 \kappa \tau^{5} \lambda^{2} \tau^{\prime} \theta^{\prime \prime}+2 \kappa^{5} \tau^{2} \lambda \lambda^{\prime} \theta^{\prime \prime}+4 \kappa^{3} \tau^{4} \lambda \lambda^{\prime} \theta^{\prime \prime}+2 \kappa \tau^{6} \lambda \lambda^{\prime} \theta^{\prime \prime}+2 \tau^{6} \theta^{\prime} \lambda \kappa^{\prime} \lambda^{\prime}}{\lambda^{3} \tau^{3}\|W\|^{5}} \\
& -\frac{2 \kappa^{5} \tau \lambda^{2} \tau^{\prime} \theta^{\prime \prime}+\kappa^{5} \tau^{2} \theta^{\prime} \lambda \lambda^{\prime \prime}+2 \kappa^{3} \tau^{4} \theta^{\prime} \lambda \lambda^{\prime \prime}+3 \kappa^{4} \theta^{\prime} \tau \theta^{\prime \prime} \lambda\|W\|}{\lambda^{3} \tau^{3}\|W\|^{5}} \\
& -\frac{3 \kappa^{2} \theta^{\prime} \tau^{3} \theta^{\prime \prime} \lambda\|W\|+3 \kappa \theta^{\prime 2} \tau^{3} \lambda \kappa^{\prime}\|W\|+\kappa^{5} \theta^{\prime 3}+\kappa^{7} \theta^{\prime}+2 \kappa^{5} \tau^{2} \theta^{\prime}+\kappa^{3} \tau^{4} \theta^{\prime}}{\lambda^{3} \tau^{3}\|W\|^{5}} \\
& -\frac{\kappa^{3} \tau^{2} \theta^{\prime 3}-3 \kappa^{6} \tau^{\prime} \lambda\|W\|-3 \kappa^{6} \tau \lambda^{\prime}\|W\|-6 \kappa^{4} \tau^{3} \lambda^{\prime}\|W\|-3 \kappa^{2} \tau^{5} \lambda^{\prime}\|W\|}{\lambda^{3} \tau^{3}\|W\|^{5}} \\
& +\frac{\kappa^{5} \tau^{2} \lambda^{2} \theta^{\prime \prime \prime}+2 \kappa^{3} \tau^{4} \lambda^{2} \theta^{\prime \prime \prime}+\kappa \tau^{6} \lambda^{2} \theta^{\prime \prime \prime}+\tau^{6} \theta^{\prime} \lambda^{2} \kappa^{\prime \prime}+2 \tau^{6} \lambda^{2} \kappa^{\prime} \theta^{\prime \prime}+2 \kappa^{5} \tau^{2} \theta^{\prime} \lambda^{\prime 2}}{\lambda^{3} \tau^{3}\|W\|^{5}} \\
& +\frac{4 \kappa^{3} \tau^{4} \theta^{\prime} \lambda^{\prime 2}+2 \kappa \tau^{6} \theta \lambda^{\prime 2}+2 \kappa^{5} \theta^{\prime} \lambda^{2} \tau^{\prime 2}+3 \kappa^{4} \theta^{\prime 2} \tau \lambda^{\prime}\|W\|+2 \kappa^{2} \tau^{3} \theta^{\prime} \lambda^{2} \kappa^{\prime} \tau^{\prime}}{\lambda^{3} \tau^{3}\|W\|^{5}} \\
& +\frac{3 \kappa^{2} \theta^{\prime 2} \tau^{3} \lambda^{\prime}\|W\|+3 \kappa^{4} \theta^{\prime 2} \lambda \tau^{\prime}\|W\|+5 \kappa^{3} \tau^{2} \theta^{\prime} \lambda^{2} \tau^{\prime 2}-3 \kappa \tau^{4} \theta^{\prime} \lambda^{2} \kappa^{\prime 2}-4 \tau^{5} \theta^{\prime} \lambda^{2} \kappa^{\prime} \tau^{\prime}}{\lambda^{3} \tau^{3}\|W\|^{5}} \\
& +\frac{6 \kappa^{3} \tau^{3} \theta^{\prime} \lambda \lambda^{\prime} \tau^{\prime}+4 \kappa \tau^{5} \theta^{\prime} \lambda \lambda^{\prime} \tau^{\prime}+2 \kappa^{5} \tau \theta^{\prime} \lambda \lambda^{\prime} \tau^{\prime}+6 \theta^{\prime 2} \kappa^{2}\|W\| \tau^{2} \lambda \tau^{\prime}}{\lambda^{3} \tau^{3}\|W\|^{5}} \\
& \left\{\begin{array}{c}
\overline{\jmath_{3}}=-\frac{5 \theta^{\prime} \kappa^{2} \lambda \tau^{2} \tau^{\prime}-3 \theta^{\prime} \kappa \lambda \tau^{3} \kappa^{\prime}-\theta^{\prime} \kappa^{3} \lambda \tau \kappa^{\prime}+2 \lambda \tau^{4} \kappa \lambda^{\prime}\|W\|+2 \lambda^{2} \tau^{3} \kappa^{\prime} \tau^{\prime}\|W\|+\kappa^{5}\|W\|}{\lambda^{3} \tau^{3}\|W\|^{3}} \\
-\frac{2 \kappa \lambda^{2} \tau^{2} \tau^{\prime 2}\|W\|-\kappa^{3} \tau^{2}\|W\|-\theta^{\prime 2} \kappa^{3}\|W\|+2 \kappa^{2} \lambda^{2} \tau \kappa^{\prime} \tau^{\prime}\|W\|+2 \kappa^{2} \lambda \tau^{2} \kappa^{\prime} \lambda^{\prime}\|W\|}{\lambda^{3} \tau^{3}\|W\|^{3}}
\end{array}\right. \\
& +\frac{2 \kappa^{2} \lambda \tau^{3} \theta^{\prime \prime}+\lambda^{2} \tau^{4} \kappa^{\prime \prime}\|W\|-3 \theta^{\prime} \kappa^{4} \lambda \tau^{\prime}-3 \theta^{\prime} \kappa^{4} \tau \lambda^{\prime}-3 \theta^{\prime} \kappa^{2} \tau^{3} \lambda^{\prime}+2 \kappa^{3} \tau^{2} \lambda^{\prime 2}\|W\|}{\lambda^{3} \tau^{3}\|W\|^{3}} \\
& +\frac{2 \kappa \tau^{4} \lambda^{\prime 2}\|W\|+2 \kappa^{3} \lambda^{2} \tau^{\prime 2}\|W\|+\kappa^{2} \lambda^{2} \tau^{2} \kappa^{\prime \prime}\|W\|+2 \kappa^{3} \lambda \tau \lambda^{\prime} \tau^{\prime}\|W\|+2 \kappa \lambda \tau^{3} \lambda^{\prime} \tau^{\prime}\|W\|}{\lambda^{3} \tau^{3}\|W\|^{3}} \\
& -\frac{\kappa^{3} \lambda \tau^{2} \lambda^{\prime \prime}\|W\|+\kappa \lambda \tau^{4} \lambda^{\prime \prime}\|W\|+\kappa^{3} \lambda^{2} \tau \tau^{\prime \prime}\|W\|+\kappa \lambda^{2} \tau^{3} \tau^{\prime \prime}\|W\|+2 \kappa^{4} \lambda \tau \theta^{\prime \prime}}{\lambda^{3} \tau^{3}\|W\|^{3}} .
\end{aligned}
$$

The torsion is then given by

$$
\tau_{\beta_{1}}=\frac{\operatorname{det}\left(\beta_{1}^{\prime}, \beta_{1}^{\prime \prime}, \beta_{1}^{\prime \prime \prime}\right)}{\left\|\beta_{1}^{\prime} \wedge \beta_{1}^{\prime \prime}\right\|^{2}}
$$




$$
\begin{aligned}
& \sqrt{2}\left[\kappa^{5} \theta^{\prime} \overline{\jmath_{3}}+\kappa^{5}\|W\| \overline{\jmath_{1}}+2 \kappa^{4} \theta \tau \lambda^{\prime} \overline{\jmath_{1}}+2 \kappa^{4} \theta^{\prime} \lambda \tau^{\prime} \overline{\jmath_{1}}-\kappa^{4} \tau \lambda \theta^{\prime \prime} \overline{\jmath_{2}}-\kappa^{4} \tau \lambda \theta^{\prime \prime} \overline{\jmath_{1}}\right. \\
& +\overline{\jmath_{2}} \kappa^{3} \theta^{\prime} \kappa^{\prime} \lambda \tau-\kappa^{3} \theta^{\prime} \tau \lambda \kappa^{\prime} \overline{\jmath_{1}}+\theta^{\prime 3} \kappa^{3} \overline{\jmath_{3}}+\kappa^{3} \theta^{\prime 2} \overline{\jmath_{1}}\|W\|+\kappa^{3} \tau^{2} \theta^{\prime} \overline{\jmath_{3}}+\kappa^{3} \theta^{\prime 2}\|W\| \\
& +\kappa^{3} \tau^{2}\|W\| \bar{\jmath}_{1}+2 \kappa^{2} \tau^{3} \theta^{\prime} \lambda^{\prime} \bar{\jmath}_{1}+\bar{\jmath}_{2} \kappa^{2} \theta^{\prime} \tau^{\prime} \lambda \tau^{2}+3 \kappa^{2} \theta^{\prime} \tau^{\prime} \lambda \tau^{2} \overline{\jmath_{1}}-\kappa^{2} \tau^{3} \lambda \theta^{\prime \prime} \overline{j_{2}} \\
& \tau_{\beta_{1}}=\frac{\left.-\kappa^{2} \tau^{3} \lambda \theta^{\prime \prime} \bar{\jmath}_{1}+\theta^{\prime 2} \kappa^{2} \lambda \tau\|W\|-2 \kappa \tau^{3} \theta^{\prime} \lambda \kappa^{\prime} \bar{\jmath}_{1}\right] \lambda^{3} \tau^{3}\|W\|^{3}}{\left[2 \kappa^{8} \tau^{2} \lambda^{2} \theta^{\prime \prime 2}+4 \kappa^{6} \lambda^{2} \tau^{4} \theta^{\prime \prime 2}+2 \kappa^{4} \lambda^{2} \tau^{6} \theta^{\prime \prime 2}+16 \kappa^{8} \tau^{2} \theta^{\prime 2}+8 \kappa^{6} \tau^{4} \theta^{\prime 2}\right.} \\
& +5 \kappa^{6} \tau^{2} \theta^{\prime 4}-4 \kappa^{7} \tau^{2} \theta^{\prime} \lambda^{2} \kappa^{\prime} \theta^{\prime \prime}-4 \kappa^{6} \tau^{3} \theta^{\prime} \lambda^{2} \tau^{\prime} \theta^{\prime \prime}-4 \kappa^{5} \tau^{4} \theta^{\prime} \lambda^{2} \kappa^{\prime} \theta^{\prime \prime}+\kappa^{6} \theta^{\prime 6} \\
& +4 \kappa^{5} \tau^{3} \theta^{\prime 2} \lambda^{2} \kappa^{\prime} \tau^{\prime}-4 \kappa^{4} \tau^{5} \theta^{\prime} \lambda^{2} \tau^{\prime} \theta^{\prime \prime}-4 \kappa^{9} \tau \theta^{\prime} \lambda \theta^{\prime \prime}-8 \kappa^{7} \tau^{3} \theta^{\prime} \lambda \theta^{\prime \prime}+4 \kappa^{12} \\
& -2 \kappa^{7} \tau \theta^{\prime 3} \lambda \theta^{\prime \prime}-4 \kappa^{5} \tau^{5} \theta^{\prime} \lambda \theta^{\prime \prime}-2 \kappa^{5} \tau^{3} \theta^{\prime 3} \lambda \theta^{\prime \prime}+2 \kappa^{6} \tau^{2} \theta^{\prime 2} \lambda^{2} \kappa^{\prime 2}+5 \kappa^{8} \theta^{\prime 4} \\
& +4 \kappa^{8} \theta^{\prime 2} \kappa^{\prime} \lambda \tau+4 \kappa^{7} \theta^{\prime 2} \tau^{\prime} \lambda \tau^{2}+4 \kappa^{6} \tau^{3} \theta^{\prime 2} \lambda \kappa^{\prime}+2 \kappa^{6} \tau \theta^{\prime 4} \lambda \kappa^{\prime}+4 \kappa^{5} \tau^{4} \theta^{\prime 2} \lambda \tau^{\prime} \\
& \left.+2 \kappa^{5} \tau^{2} \theta^{\prime 4} \lambda \tau^{\prime}+12 \kappa^{10} \tau^{2}+8 \kappa^{10} \theta^{\prime 2}+12 \kappa^{8} \tau^{4}+4 \kappa^{6} \tau^{6}+2 \kappa^{4} \tau^{4} \theta^{\prime 2} \lambda^{2} \tau^{\prime 2}\right]
\end{aligned}
$$

$N^{*} B^{*}$ - Smarandache curve can be defined by

$$
\beta_{2}(s)=\frac{1}{\sqrt{2}}\left(N^{*}+B^{*}\right)
$$

Solving the above equation by substitution of $N^{*}$ and $B^{*}$ from (2.2), we obtain

$$
\beta_{2}(s)=\frac{\sin \theta T+N+\cos \theta B}{\sqrt{2}} .
$$

The Frenet invariants of the Smarandache curve, $\beta_{2}$ are given as following:

$$
\begin{aligned}
& \mathbf{T}_{\beta_{2}}=\frac{-\kappa\left(\theta^{\prime} \cos \theta+\|W\| \sin \theta\right) T+\kappa\|W\| N+\kappa\left(\theta^{\prime} \sin \theta-\|W\| \cos \theta\right) B}{\sqrt{\kappa^{2}\left(\theta^{\prime 2}+2 \kappa^{2}+2 \tau^{2}\right)}}, \\
& \mathbf{N}_{\beta_{2}}=\frac{\left(\bar{\triangle}_{1} \cos \theta+{\overline{\triangle_{2}}}_{2} \sin \theta\right) T+\bar{\triangle}_{3} N+\left({\overline{\triangle_{2}}}_{2} \cos \theta-\bar{\triangle}_{1} \sin \theta\right) B}{\sqrt{{\overline{\triangle_{1}}}^{2}+{\overline{\triangle_{2}}}^{2}+{\overline{\triangle_{3}}}^{2}}}, \\
& \mathbf{B}_{\beta_{2}}=\frac{-\kappa\left(\theta^{\prime} \sin \theta \bar{\triangle}_{3}-\cos \theta\|W\| \bar{\triangle}_{2}-\cos \theta\|W\| \bar{\triangle}_{3}+\sin \theta\|W\| \bar{\triangle}_{1}\right)}{\sqrt{\kappa^{2}\left(\theta^{\prime 2}+2 \kappa^{2}+2{\tau^{2}}^{2}\right)\left(\bar{\triangle}_{1}{ }^{2}+{\overline{\triangle_{2}}}^{2}+{\overline{\triangle_{3}}}^{2}\right)}} \mathbf{T} \\
& +\frac{\kappa\left(-\|W\| \bar{\triangle}_{1}+\theta^{\prime} \bar{\triangle}_{2}\right)}{\sqrt{\kappa^{2}\left(\theta^{\prime 2}+2 \kappa^{2}+2 \tau^{2}\right)\left({\overline{\triangle_{1}}}^{2}+{\overline{\triangle_{2}}}^{2}+{\overline{\triangle_{3}}}^{2}\right)}} \mathbf{N} \\
& +\frac{-\kappa\left(\theta^{\prime} \cos \theta \bar{\triangle}_{3}+\cos \theta\|W\| \bar{\triangle}_{1}+\sin \theta\|W\|{\overline{\triangle_{2}}}_{2} \sin \theta\|W\| \bar{\triangle}_{3}\right)}{\sqrt{\kappa^{2}\left(\theta^{\prime 2}+2 \kappa^{2}+2 \tau^{2}\right)\left({\overline{\triangle_{1}}}^{2}+{\overline{\triangle_{2}}}^{2}+{\overline{\triangle_{3}}}^{2}\right)}} \mathbf{B}, \\
& \kappa_{\beta_{2}}=\sqrt{2} \frac{\lambda^{4} \tau^{4}\|W\|^{4}}{\kappa^{4}\left(\theta^{\prime 2}+2 \kappa^{2}+2 \tau^{2}\right)^{2}} \sqrt{{\overline{\triangle_{1}}}^{2}+{\overline{\triangle_{2}}}^{2}+{\overline{\triangle_{3}}}^{2}},
\end{aligned}
$$




$$
\begin{aligned}
& \sqrt{2}\left[2 \kappa^{5} \theta^{\prime} \overline{\hbar_{3}}-\kappa^{4} \theta^{\prime \prime} \lambda \tau \overline{\hbar_{3}}-\kappa^{4} \theta^{\prime \prime} \lambda \tau \overline{\hbar_{2}}+2 \kappa^{3} \theta^{\prime} \tau^{2} \overline{\hbar_{3}}+\kappa^{3} \theta^{\prime} \kappa^{\prime} \lambda \tau \overline{\hbar_{3}}\right. \\
& +2 \lambda^{2} \tau^{2}\|W\| \kappa^{3} \overline{\hbar_{1}}+\kappa^{3} \theta^{\prime 2}\|W\| \overline{\hbar_{1}}+\kappa^{3} \theta^{\prime} \kappa^{\prime} \lambda \tau \overline{\hbar_{2}}+\theta^{\prime 3} \kappa^{3} \overline{\hbar_{3}}+\kappa^{2} \theta^{\prime} \tau^{\prime} \lambda \tau^{2} \overline{\hbar_{3}} \\
& \tau_{\beta_{2}}=\frac{\left.-\kappa^{2} \theta^{\prime \prime} \lambda \tau^{3} \overline{\hbar_{2}}-\kappa^{2} \theta^{\prime \prime} \lambda \tau^{3} \overline{\hbar_{3}}+\kappa^{2} \theta^{\prime} \tau^{\prime} \lambda \tau^{2} \overline{\hbar_{2}}+2 \kappa \lambda^{2} \tau^{4}\|W\| \overline{\hbar_{1}}\right] \lambda^{3} \tau^{3}\|W\|^{3}}{\left[2 \lambda^{2} \tau^{2} \theta^{\prime 2} \kappa^{6} \kappa^{\prime 2}+2 \lambda^{2} \tau^{4} \theta^{\prime 2} \tau^{\prime 2} \kappa^{4}+2 \theta^{\prime 4} \kappa^{6} \lambda \tau \kappa^{\prime}+2 \theta^{\prime 4} \kappa^{5} \lambda \tau^{2} \tau^{\prime}-8 \kappa^{7} \theta^{\prime} \theta^{\prime \prime} \lambda \tau^{3}\right.}, \\
& +\theta^{6} \kappa^{6}+4 \kappa^{8} \theta^{\prime 2} \kappa^{\prime} \lambda \tau+4 \kappa^{7} \theta^{\prime 2} \tau^{\prime} \lambda \tau^{2}-4 \kappa^{9} \theta^{\prime} \theta^{\prime \prime} \lambda \tau+4 \lambda^{2} \tau^{3} \theta^{\prime 2} \kappa^{5} \kappa^{\prime} \tau^{\prime} \\
& -4 \kappa^{5} \theta^{\prime} \theta^{\prime \prime} \lambda \tau^{5}-2 \kappa^{7} \theta^{\prime 3} \theta^{\prime \prime} \lambda \tau+4 \kappa^{6} \theta^{\prime 2} \lambda \tau^{3} \kappa^{\prime}-2 \kappa^{5} \theta^{\prime 3} \theta^{\prime \prime} \lambda \tau^{3}+4 \kappa^{5} \theta^{\prime 2} \lambda \tau^{4} \tau^{\prime} \\
& -4 \kappa^{6} \lambda^{2} \tau^{3} \theta^{\prime \prime} \theta^{\prime} \tau^{\prime}-4 \kappa^{5} \lambda^{2} \tau^{4} \theta^{\prime \prime} \theta^{\prime} \kappa^{\prime}-4 \kappa^{4} \lambda^{2} \tau^{5} \theta^{\prime \prime} \theta^{\prime} \tau^{\prime}+5 \kappa^{8} \theta^{\prime 4}+8 \kappa^{10} \theta^{\prime 2} \\
& +8 \kappa^{6} \theta^{\prime 2} \tau^{4}+2 \kappa^{8} \theta^{\prime \prime 2} \lambda^{2} \tau^{2}+12 \kappa^{10} \tau^{2}+12 \kappa^{8} \tau^{4}+4 \kappa^{6} \tau^{6}+16 \kappa^{8} \theta^{\prime 2} \tau^{2} \\
& \left.+4 \kappa^{12}++2 \kappa^{4} \lambda^{2} \tau^{6} \theta^{\prime \prime 2}-4 \kappa^{7} \lambda^{2} \tau^{2} \theta^{\prime \prime} \theta^{\prime} \kappa^{\prime}+4 \kappa^{6} \lambda^{2} \tau^{4} \theta^{\prime 2}+5 \kappa^{6} \theta^{\prime 4} \tau^{2}\right]
\end{aligned}
$$

where

$$
\begin{aligned}
& \int \bar{\triangle}_{1}=\frac{-2 \lambda \theta^{\prime \prime} \kappa^{5} \tau+2 \lambda \theta^{\prime} \kappa^{4} \kappa^{\prime} \tau-2 \lambda \theta^{\prime \prime} \kappa^{3} \tau^{3}+2 \lambda \theta^{\prime} \kappa^{3} \tau^{2} \tau^{\prime}+2 \theta^{\prime} \kappa^{6}+2 \theta^{\prime} \kappa^{4} \tau^{2}+\theta^{\prime \prime \prime} \kappa^{4}}{\lambda^{4} \tau^{4}\left(\kappa^{2}+\tau^{2}\right)^{\frac{3}{2}}} \\
& \bar{\triangle}_{2}=-\frac{\lambda \theta^{\prime \prime} \theta^{\prime} \kappa^{5} \tau-\lambda \theta^{\prime 2} \kappa^{4} \kappa^{\prime} \tau+\lambda \theta^{\prime \prime} \theta^{\prime} \kappa^{3} \tau^{3}-\lambda \theta^{\prime 2} \kappa^{3} \tau^{2} \tau^{\prime}-2 \kappa^{8}-4 \kappa^{6} \tau^{2}-3 \theta^{\prime 2} \kappa^{6}}{\lambda^{4} \tau^{4}\left(\kappa^{2}+\tau^{2}\right)^{2}} \\
& -\frac{2 \kappa^{4} \tau^{4}+3{\theta^{\prime}}^{2} \kappa^{4} \tau^{2}+\theta^{\prime 4} \kappa^{4}}{\lambda^{4} \tau^{4}\left(\kappa^{2}+\tau^{2}\right)^{2}} \\
& \bar{\triangle}_{3}=\frac{-\lambda \theta^{\prime \prime} \theta^{\prime} \kappa^{5} \tau+\lambda \theta^{\prime 2} \kappa^{4} \kappa^{\prime} \tau-\lambda \theta^{\prime \prime} \theta^{\prime} \kappa^{3} \tau^{3}+\lambda \theta^{\prime 2} \kappa^{3} \tau^{2} \tau^{\prime}-2 \kappa^{8}-4 \kappa^{6} \tau^{2}-\theta^{\prime 2} \kappa^{6}}{\lambda^{4} \tau^{4}\left(\kappa^{2}+\tau^{2}\right)^{2}} \\
& -\frac{2 \kappa^{4} \tau^{4}+\theta^{\prime 2} \kappa^{4} \tau^{2}}{\lambda^{4} \tau^{4}\left(\kappa^{2}+\tau^{2}\right)^{2}} \\
& \left(\overline{\hbar_{1}}=-\frac{\theta^{\prime} \kappa^{\prime \prime} \lambda^{3} \tau^{5} \kappa^{2}+2 \theta^{\prime \prime} \kappa^{\prime} \lambda^{3} \tau^{5} \kappa^{2}-2 \theta^{\prime \prime} \kappa^{5} \lambda^{\prime} \lambda^{2} \tau^{3}-4 \theta^{\prime \prime} \kappa^{3} \lambda^{\prime} \lambda^{2} \tau^{5}-2 \theta^{\prime \prime} \kappa \lambda^{\prime} \lambda^{2} \tau^{7}}{\lambda^{4} \tau^{4}\|W\|^{5}}\right. \\
& +\frac{2 \theta^{\prime \prime} \kappa^{5} \tau^{\prime} \lambda^{3} \tau^{2}+6 \theta^{\prime \prime} \kappa^{3} \tau^{\prime} \lambda^{3} \tau^{4}+4 \theta^{\prime \prime} \kappa \tau^{\prime} \lambda^{3} \tau^{6}+\theta^{\prime} \kappa^{5} \lambda^{\prime \prime} \lambda^{2} \tau^{3}+2 \theta^{\prime} \kappa^{3} \lambda^{\prime \prime} \lambda^{2} \tau^{5}}{\lambda^{4} \tau^{4}\|W\|^{5}} \\
& +\frac{\theta^{\prime} \kappa \lambda^{\prime \prime} \lambda^{2} \tau^{7}+3 \theta^{\prime} \kappa^{3} \tau^{\prime \prime} \lambda^{3} \tau^{4}+2 \theta^{\prime} \kappa \tau^{\prime \prime} \lambda^{3} \tau^{6}-2 \theta^{\prime \prime \prime} \kappa^{3} \lambda^{3} \tau^{5}-\theta^{\prime \prime \prime} \kappa \lambda^{3} \tau^{7}-\kappa^{8} \theta^{\prime}}{\lambda^{4} \tau^{4}\|W\|^{5}} \\
& +\frac{\kappa^{6} \lambda^{2} \tau^{2} \theta^{\prime \prime}+2 \kappa^{4} \lambda^{2} \tau^{4} \theta^{\prime \prime}+\kappa^{2} \lambda^{2} \tau^{6} \theta^{\prime \prime}-2 \theta^{\prime \prime} \kappa^{\prime} \lambda^{3} \tau^{7}+\theta^{\prime 3} \kappa^{5} \lambda \tau+\theta^{\prime 3} \kappa^{3} \lambda \tau^{3}}{\lambda^{4} \tau^{4}\|W\|^{5}} \\
& -\frac{6 \theta^{\prime} \kappa \lambda^{3} \tau^{5} \tau^{\prime 2}-2 \kappa^{5} \lambda^{2} \tau^{2} \theta^{\prime} \kappa^{\prime}-5 \kappa^{3} \lambda^{2} \tau^{4} \theta^{\prime} \kappa^{\prime}+7 \kappa^{4} \lambda^{2} \tau^{3} \theta^{\prime} \tau^{\prime}-\theta^{\prime} \kappa^{5} \tau^{\prime \prime} \lambda^{3} \tau^{2}}{\lambda^{4} \tau^{4}\|W\|^{5}} \\
& 6 \\
& -\frac{4 \kappa^{2} \lambda^{2} \tau^{5} \theta^{\prime} \tau^{\prime}+5 \theta^{\prime} \kappa^{3} \lambda^{3} \tau^{3} \tau^{\prime 2}-3 \kappa \lambda^{2} \tau^{6} \theta^{\prime} \kappa+3 \kappa^{6} \lambda \tau^{2} \theta^{\prime} \lambda^{\prime}+6 \kappa^{4} \lambda \tau^{4} \theta^{\prime} \lambda^{\prime}}{\lambda^{4} \tau^{4}\|W\|^{5}} \\
& -\frac{3 \kappa^{2} \lambda \tau^{6} \theta^{\prime} \lambda^{\prime}+3 \kappa^{6} \lambda^{2} \tau \theta^{\prime} \tau^{\prime}-2 \theta^{\prime} \kappa^{\prime} \lambda^{\prime} \lambda^{2} \tau^{7}-4 \theta^{\prime} \kappa^{\prime} \tau \lambda^{3} \tau^{6}+2 \theta^{\prime} \kappa^{5} \tau^{\prime 2} \lambda^{3} \tau}{\lambda^{4} \tau^{4}\|W\|^{5}} \\
& -\frac{2 \theta^{\prime} \kappa^{5} \lambda^{\prime 2} \lambda \tau^{3}+4 \theta^{\prime} \kappa^{3} \lambda^{\prime 2} \lambda \tau^{5}+2 \theta^{\prime} \kappa \lambda^{\prime 2} \lambda \tau^{7}-3 \theta^{\prime} \kappa^{\prime 2} \lambda^{3} \tau^{5} \kappa+2 \kappa^{6} \theta^{\prime} \tau^{2}}{\lambda^{4} \tau^{4}\|W\|^{5}}
\end{aligned}
$$




$$
\begin{aligned}
& \left\{\begin{array}{l}
-\frac{\kappa^{4} \theta^{\prime} \tau^{4}+\theta^{\prime} \kappa^{\prime \prime} \lambda^{3} \tau^{7}+2 \theta^{\prime} \kappa^{2} \lambda^{3} \tau^{4} \kappa^{\prime} \tau^{\prime}-2 \theta^{\prime} \kappa^{2} \lambda^{\prime} \lambda^{2} \tau^{5} \kappa^{\prime}+6 \theta^{\prime} \kappa^{3} \lambda^{\prime} \lambda^{2} \tau^{4} \tau^{\prime}}{\lambda^{4} \tau^{4}\|W\|^{5}} \\
-\frac{4 \theta^{\prime} \kappa \lambda^{\prime} \lambda^{2} \tau^{6} \tau^{\prime}+2 \theta^{\prime} \kappa^{5} \lambda^{\prime} \tau^{\prime} \lambda^{2} \tau^{2}+\theta^{\prime \prime \prime} \kappa^{5} \lambda^{3} \tau^{3}}{\lambda^{4} \tau^{4}\|W\|^{5}}
\end{array}\right. \\
& \left\{\begin{aligned}
\overline{\hbar_{2}}= & \frac{\kappa^{5} \lambda^{\prime \prime} \lambda^{2} \tau^{3}+\kappa^{5} \tau^{\prime \prime} \lambda^{3} \tau^{2}-\kappa^{4} \kappa^{\prime \prime} \lambda^{3} \tau^{3}+2 \kappa^{3} \lambda^{\prime \prime} \lambda^{2} \tau^{5}+2 \kappa^{3} \tau^{\prime \prime} \lambda^{3} \tau^{4}-2 \kappa^{2} \kappa^{\prime \prime} \lambda^{3} \tau^{5}}{\lambda^{4} \tau^{4}\|W\|^{4}} \\
& +\frac{\kappa \tau^{\prime \prime} \lambda^{3} \tau^{6} \kappa \lambda^{\prime \prime} \lambda^{2} \tau^{7}-3 \theta^{\prime} \kappa^{2} \lambda^{2} \tau^{4} \theta^{\prime \prime}-3 \theta^{\prime} \kappa^{4} \lambda^{2} \tau^{2} \theta^{\prime \prime}-\kappa^{\prime \prime} \lambda^{3} \tau^{7}-6 \kappa^{5} \lambda^{\prime} \tau^{3}}{\lambda^{4} \tau^{4}\|W\|^{4}} \\
& +\frac{2 \kappa^{5} \lambda \tau^{3}-3 \kappa^{3} \lambda^{\prime} \tau^{5}+\kappa^{3} \lambda \tau^{5}-3 \lambda^{\prime} \kappa^{7} \tau-3 \tau^{\prime} \lambda \kappa^{7}+\lambda \kappa^{7} \tau+4 \kappa^{2} \kappa^{\prime} \lambda^{\prime} \lambda^{2} \tau^{5}}{\lambda^{4} \tau^{4}\|W\|^{4}} \\
& +\frac{4 \kappa^{2} \kappa^{\prime} \tau^{\prime} \lambda^{3} \tau^{4}-2 \kappa \lambda^{\prime} \tau^{\prime} \lambda^{2} \tau^{6}-2 \kappa^{5} \lambda^{\prime} \tau^{\prime} \lambda^{2} \tau^{2}+2 \kappa^{4} \kappa^{\prime} \lambda^{\prime} \lambda^{2} \tau^{3}+2 \kappa^{4} \kappa^{\prime} \tau^{\prime} \lambda^{3} \tau^{2}}{\lambda^{4} \tau^{4}\|W\|^{4}} \\
& -\frac{4 \kappa^{3} \lambda^{\prime} \tau^{\prime} \lambda^{2} \tau^{4}-3 \theta^{\prime 2} \kappa^{4} \lambda^{2} \tau \tau^{\prime}-3 \theta^{\prime 2} \kappa^{2} \lambda \tau^{4} \lambda^{\prime}-3 \theta^{\prime 2} \kappa^{4} \lambda \tau^{2} \lambda^{\prime}+3 \theta^{\prime 2} \kappa \lambda^{2} \tau^{4} \kappa^{\prime}}{\lambda^{4} \tau^{4}\|W\|^{4}} \\
& +\frac{6 \theta^{\prime 2} \kappa^{2} \lambda^{2} \tau^{3} \tau^{\prime}+3 \kappa^{\prime} \lambda \tau \kappa^{6}-2 \kappa^{5} \lambda^{\prime 2} \lambda \tau^{3}-2 \kappa \tau^{\prime 2} \lambda^{3} \tau^{5}+2 \kappa^{\prime} \lambda^{\prime} \lambda^{2} \tau^{7}+2 \kappa^{\prime} \tau^{\prime} \lambda^{3} \tau^{6}}{\lambda^{4} \tau^{4}\|W\|^{4}} \\
& -\frac{2 \kappa^{5} \tau^{\prime 2} \lambda^{3} \tau+6 \kappa^{5} \tau^{\prime} \lambda \tau^{2}-\kappa^{5} \theta^{2} \lambda \tau-6 \kappa^{4} \kappa \lambda \tau^{3}+4 \kappa^{3} \lambda^{\prime 2} \lambda \tau^{5}+4 \kappa^{3} \tau^{\prime 2} \lambda^{3} \tau^{3}}{\lambda^{4} \tau^{4}\|W\|^{4}} \\
& -\frac{3 \kappa^{3} \tau^{\prime} \lambda \tau^{4}-\kappa^{3} \theta^{\prime 2} \lambda \tau^{3}-3 \kappa^{2} \kappa \lambda \tau^{5}+2 \kappa \lambda^{\prime 2} \lambda \tau^{7}}{\lambda^{4} \tau^{4}\|W\|^{4}},
\end{aligned}\right. \\
& \left\{\begin{array}{r}
\overline{\bar{\hbar}_{3}}=+\frac{\tau^{\prime \prime} \lambda^{2} \kappa^{2} \tau^{3}-\kappa^{\prime \prime} \lambda^{2} \kappa^{3} \tau^{2}+\lambda^{\prime \prime} \lambda \kappa^{4} \tau^{2}+\lambda^{\prime \prime} \lambda \kappa^{2} \tau^{4}+\tau^{\prime \prime} \lambda^{2} \kappa^{4} \tau-\kappa \kappa^{\prime \prime} \lambda^{2} \tau^{4}}{\lambda^{4} \tau^{4}\|W\|^{2}} \\
+\frac{2 \kappa^{\prime} \lambda^{\prime} \lambda \kappa^{3} \tau^{2}+2 \kappa^{\prime} \tau^{\prime} \lambda^{2} \kappa^{3} \tau-2 \lambda^{\prime} \tau^{\prime} \lambda \kappa^{4} \tau-2 \lambda^{\prime} \tau^{\prime} \lambda \kappa^{2} \tau^{3}+\lambda \kappa^{5} \tau-2 \lambda^{\prime 2} \kappa^{4} \tau^{2}}{\lambda^{4} \tau^{4}\|W\|^{2}}
\end{array}\right. \\
& -\frac{2 \lambda^{\prime 2} \kappa^{2} \tau^{4}+2 \tau^{\prime 2} \lambda^{2} \kappa^{4}-\lambda \kappa^{3} \tau^{3}+3 \kappa \kappa^{\prime} \lambda^{2} \tau^{4}-2 \kappa \kappa^{\prime} \lambda^{\prime} \lambda \tau^{4}-2 \kappa \kappa^{\prime} \tau^{\prime} \lambda^{2} \tau^{3}}{\lambda^{4} \tau^{4}\|W\|^{2}} \\
& +\frac{\theta^{\prime 2} \kappa^{3} \lambda \tau-3 \kappa^{\prime} \lambda^{2} \kappa^{3} \tau^{2}+3 \lambda^{\prime} \lambda \kappa^{4} \tau^{2}+3 \lambda^{\prime} \lambda \kappa^{2} \tau^{4}+3 \tau^{\prime} \lambda^{2} \kappa^{4} \tau+3 \tau^{\prime} \lambda^{2} \kappa^{2} \tau^{3}}{\lambda^{4} \tau^{4}\|W\|^{2}} \\
& +\frac{2 \tau^{\prime 2} \lambda^{2} \kappa^{2} \tau^{2}}{\lambda^{4} \tau^{4}\|W\|^{2}}
\end{aligned}
$$

$T^{*} B^{*}$ - Smarandache curve can be defined by

$$
\beta_{3}(s)=\frac{1}{\sqrt{2}}\left(T^{*}+B^{*}\right) .
$$

Solving the above equation by substitution of $T^{*}$ and $B^{*}$ from (2.2), we obtain

$$
\beta_{3}(s)=\frac{\sin \theta T+N+\cos \theta B}{\sqrt{2}} .
$$


The Frenet invariants of the Smarandache curve, $\beta_{3}$ are given as following:

$$
\begin{aligned}
& \mathbf{T}_{\beta_{3}}=\sin \theta T+\cos \theta B, \\
& \mathbf{N}_{\beta_{3}}=\frac{\overline{o_{1}} T+\overline{o_{2}} N+\overline{o_{3}} B}{\sqrt{{\overline{o_{1}}}^{2}+{\overline{o_{1}}}^{2}+{\overline{o_{3}}}^{2}}} \\
& \mathbf{B}_{\beta_{3}}=\frac{-\overline{o_{2}} \cos \theta T+\left(\overline{o_{1}} \cos \theta-\overline{o_{3}} \sin \theta\right) N+\overline{o_{2}} \sin \theta B}{\sqrt{{\overline{o_{1}}}^{2}+{\overline{o_{1}}}^{2}+{\overline{o_{3}}}^{2}}}, \\
& \kappa_{\beta_{3}}=\frac{\sqrt{{\overline{o_{1}}}^{2}+{\overline{o_{1}}}^{2}+{\overline{o_{3}}}^{2}}}{\theta^{\prime} \lambda^{2} \tau^{2}\|W\|}, \\
& \sqrt{2}\left[\kappa^{5} \theta^{\prime} \overline{p_{3}}-2 \kappa^{5} \theta^{\prime} \overline{p_{1}}+\kappa^{5}\|W\| \overline{p_{1}}+\kappa^{3} \theta^{\prime 3} \overline{p_{3}}-2 \kappa^{3} \overline{p_{3}} \theta^{\prime 2}\|W\|\right. \\
& \tau_{\beta_{3}}=\frac{\left.+\kappa^{3} \overline{p_{1}} \theta^{\prime 2}\|W\|+\kappa^{3} \theta^{\prime} \tau^{2} \overline{p_{3}}-2 \kappa^{3} \theta^{\prime} \tau^{2} \overline{p_{1}}+\kappa^{3} \tau^{2}\|W\| \overline{p_{1}}\right] \lambda^{3} \tau^{3}\|W\|^{3}}{\left[-8 \kappa^{8}\|W\| \theta^{\prime} \tau^{2}+\kappa^{6} \theta^{\prime 6}+\kappa^{12}+\kappa^{6} \tau^{6}+3 \kappa^{10} \tau^{2}+7 \kappa^{10} \theta^{\prime 2}+3 \kappa^{8} \tau^{4}\right.}, \\
& +7 \kappa^{6} \tau^{4} \theta^{\prime 2}+7 \kappa^{8} \theta^{\prime 4}+7 \kappa^{6} \theta^{\prime 4} \tau^{2}-4 \kappa^{6}\|W\| \theta^{\prime 5}-8 \kappa^{6}\|W\| \theta^{\prime 3} \tau^{2} \\
& \left.-4 \kappa^{10}\|W\| \theta^{\prime}-4 \kappa^{6}\|W\| \theta^{\prime} \tau^{4}-8 \kappa^{8}\|W\| \theta^{\prime 3}+14 \kappa^{8} \tau^{2} \theta^{2}\right]
\end{aligned}
$$

where

$$
\begin{gathered}
\left\{\begin{array}{l}
\overline{o_{1}}=-\sqrt{2} \lambda^{2} \tau^{2} \theta^{\prime} \cos \theta\|W\|+\kappa^{2} \theta^{\prime 2} \cos \theta \\
\overline{o_{2}}=\sqrt{2} \lambda^{2} \kappa^{2} \tau^{2}+\sqrt{2} \lambda^{2} \tau^{4}-\kappa^{2} \theta^{\prime}\|W\| \\
\overline{o_{3}}=\sqrt{2} \lambda^{2} \tau^{2} \theta^{\prime} \sin \theta\|W\|-\kappa^{2} \theta^{\prime 2} \sin \theta, \\
\left\{\begin{array}{l}
\overline{p_{1}}=\frac{3 \kappa^{4} \tau \theta^{\prime 2} \lambda^{\prime}-3 \kappa^{4} \tau \theta^{\prime} \lambda^{\prime}\|W\|-3 \kappa^{4} \tau \theta^{\prime} \lambda \theta^{\prime \prime}+\kappa^{4} \tau \lambda \theta^{\prime \prime}\|W\|+3 \kappa^{4} \theta^{\prime 2} \lambda \tau^{\prime}-3 \kappa^{4} \theta^{\prime} \lambda \tau^{\prime}\|W\|}{\lambda^{3} \tau^{3}\|W\|^{4}} \\
+\frac{2 \kappa^{3} \tau \theta^{\prime}\|W\| \lambda \kappa^{\prime}+3 \kappa^{2} \tau^{3} \theta^{\prime 2} \lambda^{\prime}-3 \kappa^{2} \tau^{3} \theta^{\prime} \lambda^{\prime}\|W\|-3 \kappa^{2} \tau^{3} \theta^{\prime} \lambda \theta^{\prime \prime}+\kappa^{2} \tau^{3} \lambda \theta^{\prime \prime}\|W\|}{\lambda^{3} \tau^{3}\|W\|^{4}} \\
+\frac{6 \kappa^{2} \tau^{2} \theta^{\prime 2} \lambda \tau^{\prime}-4 \kappa^{2} \tau^{2} \theta^{\prime}\|W\| \lambda \tau^{\prime}-3 \kappa \tau^{3} \theta^{\prime 2} \lambda \kappa^{\prime}+3 \kappa \tau^{3} \theta^{\prime} \lambda \kappa^{\prime}\|W\|}{\lambda^{3} \tau^{3}\|W\|^{4}}, \\
\bar{p}_{2}=\frac{4 \lambda \tau^{5} \theta^{\prime} \tau^{\prime} \kappa \theta^{\prime}+6 \lambda \tau^{3} \theta^{\prime} \tau^{\prime} \kappa^{3} \theta^{\prime}+2 \lambda \tau \theta^{\prime} \tau^{\prime} \kappa^{5} \theta^{\prime}+2 \kappa^{\prime} \lambda^{2} \tau^{3} \tau^{\prime} \kappa^{2} \theta^{\prime}-2 \kappa^{\prime} \lambda \tau^{4} \lambda^{\prime} \kappa^{2} \theta^{\prime}}{\lambda^{3} \tau^{3}\|W\|^{5}} \\
+\frac{2 \kappa^{\prime} \lambda \tau^{4} \lambda^{\prime} \kappa^{2}\|W\|+2 \kappa^{\prime} \lambda \tau^{2} \theta^{\prime} \kappa^{4}\|W\|+4 \kappa^{\prime} \lambda^{2} \tau^{3} \tau \kappa^{2}\|W\|+2 \kappa^{\prime} \lambda^{2} \tau \tau^{\prime} \kappa^{4}\|W\|}{\lambda^{3} \tau^{3}\|W\|^{5}} \\
-\frac{2 \lambda \tau^{5} \theta^{\prime} \tau^{\prime} \kappa\|W\|+4 \lambda \tau^{3} \theta^{\prime} \tau^{\prime} \kappa^{3}\|W\|+2 \lambda \tau \theta^{\prime} \tau^{\prime} \kappa^{5}\|W\|+2 \lambda^{2} \tau^{\prime 2} \kappa^{5}\|W\|}{\lambda^{3} \tau^{3}\|W\|^{5}}
\end{array}\right.
\end{array}\right.
\end{gathered}
$$




$$
\begin{aligned}
& \left\{\begin{array}{l}
+\frac{2 \tau^{2} \theta^{\prime 2} \kappa^{5} \theta^{\prime}+2 \lambda^{2} \tau^{2} \kappa^{5} \theta^{\prime}+\tau^{2} \kappa^{3} \theta^{2}\|W\|+\lambda^{2} \tau^{6} \kappa \theta^{\prime \prime \prime}+2 \lambda^{2} \tau^{4} \kappa^{3} \theta^{\prime \prime \prime}+\lambda^{2} \tau^{2} \kappa^{5} \theta^{\prime \prime \prime}}{\lambda^{3} \tau^{3}\|W\|^{5}} \\
+\frac{\lambda^{2} \tau^{6} \kappa^{\prime \prime} \theta^{\prime}+2 \kappa^{\prime} \lambda^{2} \tau^{6} \theta^{\prime \prime}-\lambda^{2} \tau^{6} \kappa^{\prime \prime}\|W\|+\kappa^{5} \theta^{\prime 2}\|W\|-\tau^{4} \kappa^{3} \theta^{\prime}-2 \tau^{2} \kappa^{5} \theta^{\prime}}{\lambda^{3} \tau^{3}\|W\|^{5}} \\
+\frac{\tau^{4} \kappa^{3}\|W\|+2 \tau^{2} \kappa^{5}\|W\|+\tau^{2} \kappa^{3} \theta^{\prime 3}+\kappa^{5} \theta^{\prime 3}-\kappa^{7} \theta^{\prime}+\kappa^{7}\|W\|-4 \lambda^{2} \tau^{2} \tau^{\prime 2} \kappa^{3}\|W\|}{\lambda^{3} \tau^{3}\|W\|^{5}} \\
+\frac{\lambda^{2} \tau^{5} \kappa \tau^{\prime \prime}\|W\|+2 \lambda^{2} \tau^{3} \kappa^{3} \tau^{\prime \prime}\|W\|+\lambda^{2} \tau \kappa^{5} \tau^{\prime \prime}\|W\|+\lambda \tau^{6} \kappa \lambda^{\prime \prime}\|W\|+2 \lambda \tau^{4} \kappa^{3} \lambda^{\prime \prime}\|W\|}{\lambda^{3} \tau^{3}\|W\|^{5}} \\
+\frac{\lambda \tau^{2} \kappa^{5} \lambda^{\prime \prime}\|W\|+2 \kappa^{\prime} \lambda \tau^{6} \theta^{\prime}\|W\|+2 \kappa^{\prime} \lambda^{2} \tau^{5} \tau^{\prime}\|W\|-2 \lambda \tau^{6} \lambda^{\prime} \kappa \theta^{\prime \prime}-4 \lambda \tau^{4} \lambda^{\prime} \kappa^{3} \theta^{\prime \prime}}{\lambda^{3} \tau^{3}\|W\|^{5}} \\
-\frac{2 \lambda \tau^{2} \theta^{\prime} \kappa^{5} \theta^{\prime \prime}+2 \lambda^{2} \tau \tau^{\prime} \kappa^{5} \theta^{\prime \prime}+2 \kappa^{\prime} \lambda \tau^{6} \lambda^{\prime} \theta^{\prime}+\lambda \tau^{6} \kappa \lambda^{\prime \prime} \theta^{\prime}+2 \lambda \tau^{4} \kappa^{3} \lambda^{\prime \prime} \theta^{\prime}+\lambda \tau^{2} \kappa^{5} \lambda^{\prime \prime} \theta^{\prime}}{\lambda^{3} \tau^{3}\|W\|^{5}} \\
-\frac{\lambda^{2} \tau \kappa^{5} \tau^{\prime \prime} \theta^{\prime}+2 \lambda^{2} \tau^{4} \kappa^{2} \kappa^{\prime \prime}\|W\|+\lambda^{2} \tau^{2} \kappa^{4} \kappa^{\prime \prime}\|W\|+3 \theta^{\prime} \kappa \lambda^{2} \tau^{4} \kappa^{\prime 2}+2 \theta^{\prime} \kappa \lambda^{2} \tau^{5} \tau^{\prime \prime}}{\lambda^{3} \tau^{3}\|W\|^{5}} \\
+\frac{6 \theta^{\prime} \kappa \lambda^{2} \tau^{4} \tau^{\prime 2}+\theta^{\prime} \kappa^{2} \lambda^{2} \tau^{4} \kappa^{\prime \prime}-3 \theta^{\prime} \kappa^{3} \lambda^{2} \tau^{3} \tau^{\prime \prime}+5 \theta^{\prime} \kappa^{3} \lambda^{2} \tau^{2} \tau^{2}+2 \kappa^{\prime} \lambda^{2} \tau^{4} \kappa^{2} \theta^{\prime \prime}}{\lambda^{3} \tau^{3}\|W\|^{5}} \\
-\frac{6 \lambda^{2} \tau^{3} \tau^{\prime} \kappa^{3} \theta^{\prime \prime}+4 \kappa^{\prime} \lambda^{2} \tau^{5} \tau^{\prime} \theta^{\prime}+2 \lambda^{2} \tau^{4} \tau^{\prime 2} \kappa\|W\|+4 \lambda^{2} \tau^{5} \tau \kappa \theta^{\prime \prime}}{\lambda^{3} \tau^{3}\|W\|^{5}},
\end{array}\right. \\
& \left\{\begin{aligned}
\overline{p_{3}}= & \frac{2 \kappa^{4} \tau \theta^{\prime \prime} \lambda-3 \kappa^{4} \tau \theta^{\prime} \lambda^{\prime}+3 \kappa^{4} \tau \lambda^{\prime}\|W\|-3 \kappa^{4} \lambda \theta^{\prime} \tau^{\prime}+3 \kappa^{4} \lambda \tau^{\prime}\|W\|+\kappa^{3} \tau \lambda \theta^{\prime} \kappa^{\prime}}{\lambda^{3} \tau^{3}\|W\|^{3}} \\
& +\frac{2 \kappa^{2} \tau^{3} \theta^{\prime \prime} \lambda-3 \kappa^{3} \tau \lambda \kappa^{\prime}\|W\|-3 \kappa^{2} \tau^{3} \theta^{\prime} \lambda^{\prime}+3 \kappa^{2} \tau^{3} \lambda^{\prime}\|W\|-5 \kappa^{2} \tau^{2} \lambda \theta^{\prime} \tau^{\prime}}{\lambda^{3} \tau^{3}\|W\|^{3}} \\
& +\frac{3 \kappa^{2} \tau^{2} \lambda \tau^{\prime}\|W\|+3 \kappa \tau^{3} \lambda \theta^{\prime} \kappa^{\prime}-3 \kappa \tau^{3} \lambda \kappa^{\prime}\|W\|}{\lambda^{3} \tau^{3}\|W\|^{3}}
\end{aligned}\right.
\end{aligned}
$$

$T^{*} N^{*} B^{*}$ - Smarandache curve can be defined by

$$
\beta_{4}(s)=\frac{1}{\sqrt{2}}\left(T^{*}+N^{*}+B^{*}\right)
$$

Solving the above equation by substitution of $T^{*}, N^{*}$ and $B^{*}$ from (2.2), we obtain

$$
\beta_{4}(s)=\frac{(\sin \theta+\cos \theta) T+N+(\cos \theta-\sin \theta) B}{\sqrt{3}} .
$$

The Frenet invariants of the Smarandache curve, $\beta_{4}$ are given as following: 


$$
\begin{aligned}
\mathbf{T}_{\beta_{4}}= & \left(\frac{-\kappa\|W\| \sqrt{\|W\|} \sin \theta-\kappa \theta^{\prime} \sqrt{\|W\|}(\cos \theta-\sin \theta)}{\sqrt{-2 \kappa^{2}\left(\kappa^{2} \theta^{\prime}-\kappa^{2}\|W\|+\theta^{\prime} \tau^{2}-\tau^{2}\|W\|-\theta^{\prime 2}\|W\|\right)}}\right) T \\
& +\left(\frac{\kappa\|W\| \sqrt{\|W\|}}{\sqrt{-2 \kappa^{2}\left(\kappa^{2} \theta^{\prime}-\kappa^{2}\|W\|+\theta^{\prime} \tau^{2}-\tau^{2}\|W\|-\theta^{\prime 2}\|W\|\right)}}\right) N \\
& +\left(\frac{-\kappa\|W\| \sqrt{\|W\|} \cos \theta+\kappa \theta^{\prime} \sqrt{\|W\|}(\cos \theta+\sin \theta)}{\sqrt{-2 \kappa^{2}\left(\kappa^{2} \theta^{\prime}-\kappa^{2}\|W\|+\theta^{\prime} \tau^{2}-\tau^{2}\|W\|-\theta^{\prime 2}\|W\|\right)}}\right) B,
\end{aligned}
$$

$\mathbf{N}_{\beta_{4}}=\frac{\left(\overline{g_{1}} \cos \theta+\overline{g_{2}} \sin \theta\right) T+\overline{g_{3}} N+\left(g_{2} \cos \theta-g_{1} \sin \theta\right) B}{\sqrt{{\overline{g_{1}}}^{2}+{\overline{g_{2}}}^{2}+{\overline{g_{3}}}^{2}}}$,

$$
\begin{aligned}
\mathbf{B}_{\beta_{4}}= & \left(\frac{-\kappa \sqrt{\|W\|}\left(\sin \theta\|W\| \overline{g_{1}}-\cos \theta\|W\|\left(\overline{g_{2}}+\overline{g_{3}}\right)+\theta^{\prime} \overline{g_{3}}(\sin \theta+\cos \theta)\right)}{\sqrt{-2 \kappa^{2}\left({\overline{g_{1}}}^{2}+{\overline{g_{2}}}^{2}+{\overline{g_{3}}}^{2}\right)\left(\kappa^{2} \theta^{\prime}-\kappa^{2}\|W\|+\theta^{\prime} \tau^{2}-\tau^{2}\|W\|-\theta^{\prime 2}\|W\|\right)}}\right) \mathbf{T} \\
& +\left(\frac{-\|W\| \overline{g_{1}}+\theta^{\prime} \overline{g_{1}}+\theta^{\prime} \overline{g_{2}}}{\sqrt{-2 \kappa^{2}\left({\overline{g_{1}}}^{2}+{\overline{g_{2}}}^{2}+{\overline{g_{3}}}^{2}\right)\left(\kappa^{2} \theta^{\prime}-\kappa^{2}\|W\|+\theta^{\prime} \tau^{2}-\tau^{2}\|W\|-\theta^{\prime 2}\|W\|\right)}}\right) \mathbf{N} \\
& +\left(\frac{\kappa \sqrt{\|W\|}\left(-\cos \theta\|W\| \overline{g_{1}}-\sin \theta\|W\|\left(\overline{g_{2}}+\overline{g_{3}}\right)+\theta^{\prime} \overline{g_{3}}(\sin \theta-\cos \theta)\right)}{\sqrt{-2 \kappa^{2}\left(\bar{g}_{1}^{2}+\bar{g}_{2}^{2}+\bar{g}_{3}^{2}\right)\left(\kappa^{2} \theta^{\prime}-\kappa^{2}\|W\|+\theta^{\prime} \tau^{2}-\tau^{2}\|W\|-\theta^{\prime 2}\|W\|\right)}}\right) \mathbf{B}, \\
\kappa_{\beta_{4}}= & \frac{\sqrt{3} \lambda^{8} \tau^{8}\|W\|^{4} \sqrt{{\overline{g_{1}}}^{2}+{\overline{g_{2}}}^{2}+{\overline{g_{3}}}^{2}}}{2 \kappa^{4}\left(\kappa^{2} \tau^{2} \lambda^{2}+\tau^{4} \lambda^{2}+{\theta^{\prime}}^{2} \lambda^{2} \tau^{2}-{\theta^{\prime}}^{2} \kappa^{2}\right)^{2}},
\end{aligned}
$$

$$
\begin{aligned}
& \sqrt{3}\left[2 \kappa^{5} \theta^{\prime} \bar{f}_{3}-2 \kappa^{5} \theta^{\prime} \bar{f}_{1}+2 \kappa^{5}\|W\| \bar{f}_{1}-\kappa^{4} \tau \lambda \theta^{\prime \prime} \bar{f}_{2}-\kappa^{4} \tau \lambda \theta^{\prime \prime} \bar{f}_{3}-\kappa^{2} \tau^{3} \lambda \theta^{\prime \prime} \bar{f}_{2}-\kappa^{2} \tau^{3} \lambda \theta^{\prime \prime} \bar{f}_{3}\right. \\
& -\kappa^{4} \tau \lambda \theta^{\prime \prime} \bar{f}_{1}+\bar{f}_{2} \kappa^{3} \tau \theta^{\prime} \lambda \kappa^{\prime}+\bar{f}_{3} \kappa^{3} \tau \theta^{\prime} \lambda \kappa^{\prime}+\bar{f}_{1} \kappa^{3} \tau \theta^{\prime} \lambda \kappa^{\prime}+2 \kappa^{3} \tau^{2} \theta^{\prime} \bar{f}_{3}-\kappa^{2} \tau^{3} \lambda \theta^{\prime \prime} \bar{f}_{1} \\
& +\bar{f}_{3} \kappa^{2} \tau^{2} \theta^{\prime} \lambda \tau^{\prime}+\bar{f}_{1} \kappa^{2} \tau^{2} \theta^{\prime} \lambda \tau^{\prime}+\bar{f}_{2} \kappa^{2} \tau^{2} \theta^{\prime} \lambda \tau^{\prime}-2 \kappa^{3} \tau^{2} \theta^{\prime} \bar{f}_{1}+2 \kappa^{3} \tau^{2}\|W\| \bar{f}_{1}+2 \theta^{\prime 3} \kappa^{3} \bar{f}_{3} \\
\tau_{\beta_{4}}= & \left.-2 \kappa^{3} \theta^{\prime 2} \bar{f}_{3}\|W\|+2 \kappa^{3} \theta^{\prime 2} \bar{f}_{1}\|W\|\right] \lambda^{3} \tau^{3}\|W\|^{3} \\
& {\left[-16 \kappa^{8} \theta^{\prime 3}\|W\|-8 \kappa^{10} \theta^{\prime}\|W\|+16 \kappa^{6} \tau^{2} \theta^{\prime 4}+32 \kappa^{8} \tau^{2} \theta^{\prime 2}+16 \kappa^{6} \tau^{4} \theta^{\prime 2}-8 \kappa^{6} \theta^{\prime 5}\|W\|\right.} \\
& +4 \kappa^{12}+16 \kappa^{8} \theta^{\prime 2}+16 \kappa^{10} \theta^{\prime 2}+12 \kappa^{10} \tau^{2}+12 \kappa^{8} \tau^{4}+4 \kappa^{6} \tau^{6}+4 \kappa^{6} \theta^{\prime 6}-4 \kappa^{9} \tau \lambda \theta^{\prime \prime}\|W\| \\
& -8 \kappa^{7} \tau^{3} \lambda \theta^{\prime \prime}\|W\|-4 \kappa^{5} \tau^{5} \lambda \theta^{\prime \prime}\|W\|+3 \kappa^{4} \tau^{4} \theta^{\prime 2} \lambda^{2} \tau^{\prime 2}-4 \kappa^{7} \tau \theta^{\prime 3} \lambda \theta^{\prime}-4 \kappa^{5} \tau^{3} \theta^{\prime 3} \lambda \theta^{\prime \prime} \\
& +3 \kappa^{6} \tau^{2} \theta^{\prime 2} \lambda^{2} \kappa^{\prime 2}+4 \kappa^{6} \tau \theta^{\prime \prime} \lambda \kappa^{\prime}+4 \kappa^{5} \tau^{2} \theta^{\prime 4} \lambda \tau^{\prime}+4 \kappa^{8} \tau \theta^{\prime} \lambda \kappa^{\prime}\|W\|+4 \kappa^{6} \tau^{3} \theta^{\prime} \lambda \kappa^{\prime}\|W\| \\
& +4 \kappa^{7} \tau^{2} \theta^{\prime} \lambda \tau^{\prime}\|W\|+4 \kappa^{5} \tau^{4} \theta^{\prime} \lambda \tau^{\prime}\|W\|-6 \kappa^{6} \tau^{3} \theta^{\prime} \lambda^{2} \tau^{\prime} \theta^{\prime \prime}-6 \kappa^{7} \tau^{2} \theta^{\prime} \lambda^{2} \kappa^{\prime} \theta^{\prime \prime}+3 \kappa^{8} \tau^{2} \lambda^{2} \theta^{\prime \prime 2} \\
& +6 \kappa^{5} \tau^{3} \theta^{\prime 2} \lambda^{2} \kappa^{\prime} \tau^{\prime}-6 \kappa^{4} \tau^{5} \theta^{\prime} \lambda^{2} \tau^{\prime} \theta^{\prime \prime}-16 \kappa^{6} \tau^{2} \theta^{\prime 3}\|W\|-16 \kappa^{8} \tau^{2} \theta^{\prime}\|W\|-8 \kappa^{6} \tau^{4} \theta^{\prime}\|W\| \\
& \left.-6 \kappa^{5} \tau^{4} \theta^{\prime} \lambda^{2} \kappa^{\prime} \theta^{\prime \prime}+6 \kappa^{6} \tau^{4} \lambda^{2} \theta^{\prime \prime 2}+3 \kappa^{4} \tau^{6} \lambda^{2} \theta^{\prime \prime 2}\right]
\end{aligned}
$$

where 


$$
\begin{aligned}
& \int \overline{g_{1}}=\frac{\kappa^{3} \tau^{3} \theta^{\prime} \lambda \theta^{\prime \prime}-2 \kappa^{3} \tau^{3} \lambda \theta^{\prime \prime}\|W\|-\theta^{\prime 2} \kappa^{3} \tau^{\prime} \lambda \tau^{2}+2 \kappa^{3}\|W\|-\tau^{2} \theta^{\prime} \lambda \tau^{\prime}-\theta^{\prime 2} \kappa^{4} \kappa^{\prime} \lambda \tau}{\lambda^{4} \tau^{4}\|W\|^{4}} \\
& +\frac{\kappa^{5} \tau \theta^{\prime} \lambda \theta^{\prime \prime}+2 \kappa^{4} \tau \theta^{\prime} \lambda\|W\|-\kappa^{\prime}-2 \kappa^{5} \tau \lambda \theta^{\prime \prime}\|W\|-4 \kappa^{4} \tau^{2} \theta^{\prime 2}+2 \kappa^{4} \tau^{2} \theta^{\prime}\|W\|}{\lambda^{4} \tau^{4}\|W\|^{4}} \\
& -\frac{2 \theta^{\prime 4} \kappa^{4}-4 \kappa^{4} \theta^{\prime 3}\|W\|+4 \kappa^{6} \theta^{\prime 2}-2 \kappa^{6} \theta^{\prime}\|W\|}{\lambda^{4} \tau^{4}\|W\|^{4}} \\
& \overline{g_{2}}=\frac{\kappa^{3} \tau^{3} \theta^{\prime} \lambda \theta^{\prime \prime}+\kappa^{3} \tau^{3} \lambda \theta^{\prime \prime}\|W\|-\theta^{\prime 2} \kappa^{3} \tau^{\prime} \lambda \tau^{2}-\kappa^{3}\|W\| \tau^{2} \theta^{\prime} \lambda \tau^{\prime}-\theta^{\prime 2} \kappa^{4} \kappa^{\prime} \lambda \tau-2 \kappa^{8}}{\lambda^{4} \tau^{4}\|W\|^{4}} \\
& -\frac{\kappa^{4} \tau \theta^{\prime} \lambda\|W\| \kappa^{\prime}-\kappa^{5} \tau \lambda \theta^{\prime \prime}\|W\|+2 \kappa^{4} \tau^{4}+4 \kappa^{4} \tau^{2} \theta^{\prime 2}-2 \kappa^{4} \tau^{2} \theta^{\prime}\|W\|-\kappa^{5} \tau \theta^{\prime} \lambda \theta^{\prime \prime}}{\lambda^{4} \tau^{4}\|W\|^{4}} \\
& -\frac{4 \kappa^{6} \tau^{2}+2 \theta^{\prime 4} \kappa^{4}-2 \kappa^{4} \theta^{\prime 3}\|W\|+4 \kappa^{6} \theta^{\prime 2}-2 \kappa^{6} \theta^{\prime}\|W\|}{\lambda^{4} \tau^{4}\|W\|^{4}} \\
& \overline{g_{3}}=\frac{\kappa^{3} \tau^{3} \lambda \theta^{\prime \prime}\|W\|-2 \kappa^{3} \tau^{3} \theta^{\prime} \lambda \theta^{\prime \prime}+2 \theta^{\prime 2} \kappa^{3} \tau^{\prime} \lambda \tau^{2}-\kappa^{3}\|W\| \tau^{2} \theta^{\prime} \lambda \tau^{\prime}+2 \theta^{\prime 2} \kappa^{4} \kappa^{\prime} \lambda \tau}{\lambda^{4} \tau^{4}\|W\|^{4}} \\
& -\frac{\kappa^{4} \tau \theta^{\prime} \lambda\|W\| \kappa^{\prime}-\kappa^{5} \tau \lambda \theta^{\prime \prime}\|W\|+2 \kappa^{4} \tau^{4}+4 \kappa^{4} \tau^{2} \theta^{\prime 2}-4 \kappa^{4} \tau^{2} \theta^{\prime}\|W\|+4 \kappa^{6} \tau^{2}}{\lambda^{4} \tau^{4}\|W\|^{4}} \\
& +\frac{2 \kappa^{4} \theta^{\prime 3}\|W\|-4 \kappa^{6} \theta^{\prime 2}+4 \kappa^{6} \theta^{\prime}\|W\|-2 \kappa^{8}-2 \kappa^{5} \tau \theta^{\prime} \lambda \theta^{\prime \prime}}{\lambda^{4} \tau^{4}\|W\|^{4}}, \\
& \left\{\begin{array}{c}
\bar{f}_{1}=-\frac{\kappa^{2} \tau^{4} \theta^{\prime} \lambda^{2} \kappa^{\prime \prime}+7 \kappa^{4} \tau^{2} \theta^{\prime} \lambda \tau^{\prime}+4 \kappa^{2} \tau^{4} \theta^{\prime} \lambda \tau^{\prime}+2 \kappa^{2} \tau^{4} \lambda^{2} \kappa^{\prime} \theta^{\prime \prime}+5 \kappa^{3} \tau^{2} \theta^{\prime} \lambda^{2} \tau^{\prime 2}}{\lambda^{3} \tau^{3}\|W\|^{5}} \\
+\frac{2 \kappa^{5} \tau \theta^{\prime} \lambda \kappa^{\prime}+5 \kappa^{3} \tau^{3} \theta^{\prime} \lambda \kappa^{\prime}+3 \kappa \tau^{5} \theta^{\prime} \lambda \kappa^{\prime}+6 \kappa^{3} \tau^{3} \lambda^{2} \tau^{\prime} \theta^{\prime \prime}+4 \kappa \tau^{5} \lambda^{2} \tau^{\prime} \theta^{\prime \prime}+\kappa^{5} \theta^{\prime 3}}{\lambda^{3} \tau^{3}\|W\|^{5}}
\end{array}\right. \\
& +\frac{4 \tau^{5} \theta^{\prime} \lambda^{2} \kappa^{\prime} \tau^{\prime}+2 \kappa^{5} \tau^{2} \lambda \lambda^{\prime} \theta^{\prime}+4 \kappa^{3} \tau^{4} \lambda \lambda^{\prime} \theta^{\prime \prime}+2 \kappa \tau^{6} \lambda \lambda^{\prime} \theta^{\prime \prime}+2 \kappa^{5} \tau \lambda^{2} \tau^{\prime} \theta^{\prime \prime}+\kappa^{7} \theta^{\prime}}{\lambda^{3} \tau^{3}\|W\|^{5}} \\
& +\frac{2 \tau^{6} \theta^{\prime} \lambda \lambda^{\prime} \kappa^{\prime}+\kappa^{5} \tau^{2} \theta^{\prime} \lambda \lambda^{\prime \prime}+2 \kappa^{3} \tau^{4} \theta^{\prime} \lambda \lambda^{\prime \prime}+\kappa \tau^{6} \theta^{\prime} \lambda \lambda^{\prime \prime}+\kappa^{5} \tau \theta^{\prime} \lambda^{2} \tau^{\prime \prime}+2 \kappa^{2} \tau^{4} \theta^{\prime} \lambda \lambda^{\prime} \kappa^{\prime}}{\lambda^{3} \tau^{3}\|W\|^{5}} \\
& +\frac{3 \kappa^{3} \tau^{3} \theta^{\prime} \lambda^{2} \tau^{\prime \prime}+2 \kappa \tau^{5} \theta^{\prime} \lambda^{2} \tau^{\prime \prime}+3 \kappa \tau^{4} \theta^{\prime} \lambda^{2} \kappa^{\prime 2}-6 \kappa \tau^{4} \theta^{\prime} \lambda^{2} \tau^{\prime 2}+6 \theta^{\prime 2} \kappa^{2}\|W\| \tau^{2} \lambda \tau^{\prime}}{\lambda^{3} \tau^{3}\|W\|^{5}} \\
& -\frac{2 \kappa^{2} \tau^{3} \theta^{\prime} \lambda^{2} \kappa^{\prime} \tau^{\prime}-\kappa^{3} \tau^{2} \theta^{\prime 3}-2 \kappa^{5} \tau^{2} \theta^{\prime}-\kappa^{3} \tau^{4} \theta^{\prime}+\kappa^{5} \tau^{2} \lambda^{2} \theta^{\prime \prime \prime}+3 \kappa \tau^{3} \theta^{\prime 2} \lambda \kappa^{\prime}\|W\|}{\lambda^{3} \tau^{3}\|W\|^{5}} \\
& -\frac{2 \kappa^{3} \tau^{4} \lambda^{2} \theta^{\prime \prime \prime}+\kappa \tau^{6} \lambda^{2} \theta^{\prime \prime \prime}+\tau^{6} \theta^{\prime} \lambda^{2} \kappa^{\prime \prime}+3 \kappa^{6} \tau \theta^{\prime} \lambda^{\prime}+6 \kappa^{4} \tau^{3} \theta^{\prime} \lambda^{\prime}+2 \kappa^{5} \tau \theta^{\prime} \lambda \lambda^{\prime} \tau^{\prime}}{\lambda^{3} \tau^{3}\|W\|^{5}}
\end{aligned}
$$




$$
\begin{aligned}
& \left\{\begin{array}{c}
-\frac{3 \kappa^{2} \tau^{5} \theta^{\prime} \lambda^{\prime}+3 \kappa^{6} \theta^{\prime} \lambda \tau^{\prime}-\kappa^{6} \tau \lambda \theta^{\prime \prime}-2 \kappa^{4} \tau^{3} \lambda \theta^{\prime \prime}+6 \kappa^{3} \tau^{3} \theta^{\prime} \lambda \lambda^{\prime} \tau^{\prime}+3 \kappa^{2} \tau^{3} \theta^{\prime} \lambda \theta^{\prime \prime}\|W\|}{\lambda^{3} \tau^{3}\|W\|^{5}} \\
-\frac{2 \tau^{6} \lambda^{2} \kappa^{\prime} \theta^{\prime \prime}+2 \kappa^{5} \tau^{2} \theta^{\prime} \lambda^{\prime 2}+4 \kappa^{3} \tau^{4} \theta^{\prime} \lambda^{\prime 2}+2 \kappa \tau^{6} \theta^{\prime} \lambda^{\prime 2}+2 \kappa^{5} \theta^{\prime} \lambda^{2} \tau^{\prime 2}+4 \kappa \tau^{5} \theta^{\prime} \lambda \lambda^{\prime} \tau^{\prime}}{\lambda^{3} \tau^{3}\|W\|^{5}} \\
+\frac{3 \kappa^{4} \tau \theta^{\prime 2} \lambda^{\prime}\|W\|+3 \kappa^{2} \tau^{3} \theta^{\prime 2} \lambda^{\prime}\|W\|+3 \kappa^{4} \theta^{\prime 2} \lambda \tau^{\prime}\|W\|-3 \kappa^{4} \tau \theta^{\prime} \lambda \theta^{\prime \prime}\|W\|+\kappa^{2} \tau^{5} \lambda \theta^{\prime \prime}}{\lambda^{3} \tau^{3}\|W\|^{5}}
\end{array}\right.
\end{aligned}
$$

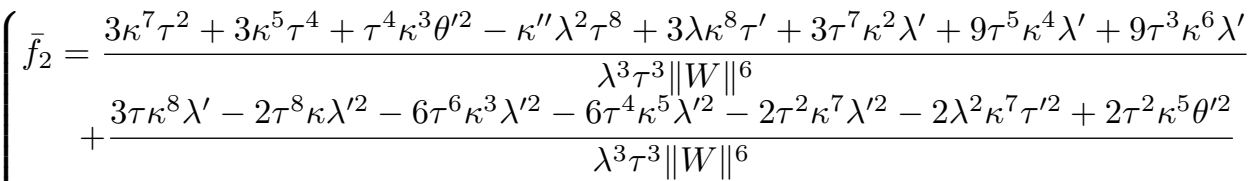

$$
\begin{aligned}
& -\frac{+\kappa^{7} \theta^{\prime}\|W\|+\kappa^{5} \theta^{\prime 3}\|W\|-\tau^{6} \kappa^{3}-\kappa^{7} \theta^{\prime 2}-6 \lambda \tau^{6} \kappa^{2} \kappa^{\prime} \lambda^{\prime}-6 \lambda^{2} \tau^{5} \kappa^{2} \kappa^{\prime} \tau^{\prime}+2 \lambda \tau^{7} \kappa \tau^{\prime} \lambda^{\prime}}{\lambda^{3} \tau^{3}\|W\|^{6}} \\
& +\frac{6 \lambda^{2} \tau^{3} \kappa^{4} \kappa^{\prime} \tau^{\prime}-6 \lambda \tau^{5} \kappa^{3} \tau^{\prime} \lambda^{\prime}-6 \lambda \tau^{3} \kappa^{5} \tau^{\prime} \lambda^{\prime}-2 \lambda \tau \kappa^{7} \tau^{\prime} \lambda^{\prime}+6 \lambda \tau^{4} \kappa^{4} \kappa^{\prime} \lambda^{\prime}+2 \lambda \tau^{2} \kappa^{6} \kappa^{\prime} \lambda^{\prime}}{\lambda^{3} \tau^{3}\|W\|^{6}} \\
& +\frac{2 \lambda^{2} \tau \kappa^{6} \kappa^{\prime} \tau^{\prime}-3 \theta^{\prime} \kappa^{6} \lambda \tau \theta^{\prime \prime}+9 \theta^{\prime 2} \kappa^{4} \lambda \tau^{2} \tau^{\prime}-3 \theta^{\prime 2} \kappa^{3} \lambda \tau^{3} \kappa^{\prime}-6 \theta^{\prime} \kappa^{4} \lambda \tau^{3} \theta^{\prime \prime}+6 \theta^{\prime 2} \kappa^{2} \lambda \tau^{4} \tau^{\prime}}{\lambda^{3} \tau^{3}\|W\|^{6}} \\
& +\frac{2 \tau^{2} \kappa^{5} \theta^{\prime} \lambda^{\prime 2}\|W\|-3 \theta^{\prime 2} \kappa \lambda \tau^{5} \kappa^{\prime}-3 \theta^{\prime} \kappa^{2} \lambda \tau^{5} \theta^{\prime}+2 \tau^{6} \kappa \theta^{\prime} \lambda^{\prime 2}\|W\|+4 \tau^{4} \kappa^{3} \theta^{\prime} \lambda^{\prime 2}\|W\|}{\lambda^{3} \tau^{3}\|W\|^{6}} \\
& +\frac{2 \lambda^{2} \kappa^{5} \theta^{\prime} \tau^{\prime 2}\|W\|+\kappa^{\prime \prime} \lambda^{2} \tau^{6} \theta^{\prime}\|W\|+2 \lambda^{2} \tau^{6} \kappa \theta^{\prime \prime}\|W\|+\lambda^{2} \tau^{6} \kappa \theta^{\prime \prime \prime}\|W\|+2 \lambda^{2} \tau^{4} \kappa^{3} \theta^{\prime \prime \prime}\|W\|}{\lambda^{3} \tau^{3}\|W\|^{6}} \\
& +\frac{\lambda^{2} \tau^{2} \kappa^{5} \theta^{\prime \prime \prime}\|W\|+2 \lambda^{2} \tau^{3} \kappa^{2} \theta^{\prime} \kappa^{\prime} \tau^{\prime}\|W\|+2 \lambda \tau \kappa^{5} \theta^{\prime} \tau^{\prime} \lambda^{\prime}\|W\|+4 \theta^{\prime} \kappa \lambda^{\prime} \lambda \tau^{5}\|W\| \tau^{\prime}}{\lambda^{3} \tau^{3}\|W\|^{6}} \\
& +\frac{6 \theta^{\prime} \kappa^{3} \lambda^{\prime} \lambda \tau^{3}\|W\| \tau^{\prime}-2 \theta^{\prime} \kappa^{2} \lambda^{\prime} \lambda \tau^{4}\|W\| \kappa^{\prime}+\theta^{\prime} \kappa^{2} \lambda^{2} \tau^{4}\|W\| \kappa^{\prime \prime}-2 \theta^{\prime} \kappa \lambda^{2} \tau^{5}\|W\| \tau^{\prime \prime}}{\lambda^{3} \tau^{3}\|W\|^{6}} \\
& +\frac{6 \theta^{\prime} \kappa \lambda^{2} \tau^{4}\|W\| \tau^{\prime 2}-3 \theta^{\prime} \kappa \lambda^{2} \tau^{4}\|W\| \kappa^{\prime 2}-3 \theta^{\prime} \kappa^{3} \lambda^{2} \tau^{3}\|W\| \tau^{\prime \prime}+5 \theta^{\prime} \kappa^{3} \lambda^{2} \tau^{2}\|W\| \tau^{\prime 2}}{\lambda^{3} \tau^{3}\|W\|^{6}} \\
& -\frac{4 \lambda \tau^{4} \kappa^{3} \lambda^{\prime} \theta^{\prime \prime}\|W\|+2 \lambda \tau^{2} \kappa^{5} \lambda^{\prime} \theta^{\prime \prime}\|W\|+4 \lambda^{2} \tau^{5} \kappa \tau^{\prime} \theta^{\prime \prime}\|W\|+6 \lambda^{2} \tau^{3} \kappa^{3} \tau^{\prime} \theta^{\prime \prime}\|W\|}{\lambda^{3} \tau^{3}\|W\|^{6}} \\
& -\frac{4 \lambda^{2} \tau^{5} \theta^{\prime} \kappa^{\prime} \tau^{\prime}\|W\|+\lambda \tau^{6} \kappa \theta^{\prime} \lambda^{\prime \prime}\|W\|+2 \lambda \tau^{4} \kappa^{3} \theta^{\prime} \lambda^{\prime}\|W\|+\lambda \tau^{2} \kappa^{5} \theta^{\prime} \lambda^{\prime \prime}\|W\|}{\lambda^{3} \tau^{3}\|W\|^{6}} \\
& +\frac{2 \lambda^{2} \tau^{4} \kappa^{2} \kappa^{\prime} \theta^{\prime \prime}\|W\|+2 \lambda^{2} \tau^{7} \kappa^{\prime} \tau^{\prime}-3 \kappa^{\prime \prime} \lambda^{2} \tau^{6} \kappa^{2}-3 \kappa^{\prime \prime} \lambda^{2} \tau^{4} \kappa^{4}-\kappa^{\prime \prime} \lambda^{2} \tau^{2} \kappa^{6}}{\lambda^{3} \tau^{3}\|W\|^{6}} \\
& +\frac{\lambda^{2} \tau \kappa^{7} \tau^{\prime \prime}+\lambda \tau^{8} \kappa \lambda^{\prime \prime}+3 \lambda \tau^{4} \kappa^{5} \lambda^{\prime \prime}+\lambda \tau^{2} \kappa^{7} \lambda^{\prime \prime}+\lambda^{2} \tau^{7} \kappa \tau^{\prime \prime}+3 \lambda^{2} \tau^{5} \kappa^{3} \tau^{\prime \prime}+3 \lambda^{2} \tau^{3} \kappa^{5} \tau^{\prime \prime}}{\lambda^{3} \tau^{3}\|W\|^{6}} \\
& +\frac{9 \lambda \tau^{2} \kappa^{6} \tau^{\prime}-3 \lambda \tau \kappa^{7} \kappa^{\prime}-2 \lambda^{2} \tau^{6} \kappa \tau^{\prime 2}-6 \lambda^{2} \tau^{4} \kappa^{3} \tau^{\prime 2}-6 \lambda^{2} \tau^{2} \kappa^{5} \tau^{\prime 2}+2 \lambda \tau^{8} \kappa^{\prime} \lambda^{\prime}+3 \theta^{\prime 2} \kappa^{6} \tau \lambda^{\prime}}{\lambda^{3} \tau^{3}\|W\|^{6}} \\
& +\frac{3 \theta^{\prime 2} \kappa^{6} \lambda \tau^{\prime}-\tau^{2}+6 \theta^{\prime 2} \kappa^{4} \tau^{3} \lambda^{\prime}+3 \theta^{\prime 2} \kappa^{2} \tau^{5} \lambda^{\prime} \kappa^{3} \theta^{\prime 3}\|W\|-\tau^{4} \kappa^{3} \theta^{\prime}\|W\|-2 \tau^{2} \kappa^{5} \theta^{\prime}\|W\|+\kappa^{9}}{\lambda^{3} \tau^{3}\|W\|^{6}} \\
& +\frac{3 \lambda \tau^{6} \kappa^{2} \tau^{\prime}-9 \lambda \tau^{5} \kappa^{3} \kappa^{\prime}+9 \lambda \tau^{4} \kappa^{4} \tau^{\prime}-9 \lambda \tau^{3} \kappa^{5} \kappa^{\prime}+2 \lambda^{2} \tau \kappa^{5} \tau^{\prime} \theta^{\prime \prime}\|W\|+2 \lambda \tau^{6} \theta^{\prime} \kappa^{\prime} \lambda^{\prime}\|W\|}{\lambda^{3} \tau^{3}\|W\|^{6}} \\
& +\frac{3 \lambda \tau^{6} \kappa^{3} \lambda^{\prime \prime}-3 \lambda \tau^{7} \kappa \kappa^{\prime}-2 \lambda \tau^{6} \kappa \lambda^{\prime} \theta^{\prime \prime}\|W\|+\lambda^{2} \tau \kappa^{5} \theta^{\prime} \tau^{\prime \prime}\|W\|}{\lambda^{3} \tau^{3}\|W\|^{6}},
\end{aligned}
$$




$$
\left\{\begin{aligned}
\bar{f}_{3}= & -\frac{2 \lambda \tau^{4} \kappa^{\prime} \lambda^{\prime}\|W\|+2 \lambda^{2} \tau^{3} \kappa^{\prime} \tau^{\prime}\|W\|+\kappa^{3} \lambda \tau^{2} \lambda^{\prime \prime}\|W\|+\kappa \lambda \tau^{4} \lambda^{\prime \prime}\|W\|}{\lambda^{3} \tau^{3}\|W\|^{3}} \\
& +\frac{2 \kappa \lambda^{2} \tau^{2} \tau^{\prime 2}\|W\|-\kappa^{3} \lambda^{2} \tau \tau^{\prime \prime}\|W\|-\kappa \lambda^{2} \tau^{3} \tau^{\prime \prime}\|W\|+3 \kappa^{4} \lambda \tau^{\prime}\|W\|}{\lambda^{3} \tau^{3}\|W\|^{3}} \\
& +\frac{3 \kappa^{4} \tau \lambda^{\prime}\|W\|+3 \kappa^{2} \tau^{3} \lambda^{\prime}\|W\|+\lambda^{2} \tau^{4} \kappa^{\prime \prime}\|W\|+2 \kappa^{3} \tau^{2} \lambda^{\prime 2}\|W\|}{\lambda^{3} \tau^{3}\|W\|^{3}} \\
& +\frac{2 \kappa \tau^{4} \lambda^{\prime 2}\|W\|+2 \kappa^{3} \lambda^{2} \tau^{\prime 2}\|W\|-\kappa^{3} \theta^{\prime 2}\|W\|-3 \kappa \lambda \tau^{3} \kappa^{\prime}\|W\|-\kappa^{5}\|W\|}{\lambda^{3} \tau^{3}\|W\|^{3}} \\
& +\frac{2 \kappa^{4} \lambda \tau \theta^{\prime \prime}-3 \kappa^{4} \theta^{\prime} \tau \lambda^{\prime}-3 \kappa^{2} \tau^{3} \theta^{\prime} \lambda^{\prime}+2 \kappa^{2} \tau^{3} \lambda \theta^{\prime \prime}-3 \kappa^{4} \theta^{\prime} \lambda \tau^{\prime}-\kappa^{3} \tau^{2}\|W\|}{\lambda^{3} \tau^{3}\|W\|^{3}} \\
& +\frac{3 \kappa \tau^{3} \theta^{\prime} \lambda \kappa^{\prime}-5 \tau^{2} \kappa^{2} \theta^{\prime} \lambda \tau^{\prime}+\theta^{\prime} \kappa^{3} \lambda \tau \kappa^{\prime}-3 \kappa^{3} \lambda \tau \kappa^{\prime}\|W\|+3 \kappa^{2} \lambda \tau^{2} \tau^{\prime}\|W\|}{\lambda^{3} \tau^{3}\|W\|^{3}}
\end{aligned}\right.
$$

\section{Example}

Let us consider the unit speed Mannheim curve and Mannheim partner curve:

$$
\alpha(s)=\frac{1}{\sqrt{2}}(-\cos s,-\sin s, s) \text { and } \alpha^{*}(s)=\frac{1}{\sqrt{2}}(-2 \cos s,-2 \sin s, s) .
$$

The Frenet invariants of the partner curve, $\alpha^{*}(s)$ are given as following:

$$
\left\{\begin{array}{l}
T^{*}(s)=\frac{1}{\sqrt{5}}(2 \sin s,-2 \cos s, 1), \quad N^{*}(s)=\frac{1}{\sqrt{5}}(\sin s, \cos s,-2), \quad B^{*}(s)=(\cos s, \sin s, 0) \\
\kappa^{*}(s)=\frac{2 \sqrt{2}}{5}, \quad \tau^{*}(s)=\frac{\sqrt{2}}{5}
\end{array}\right.
$$

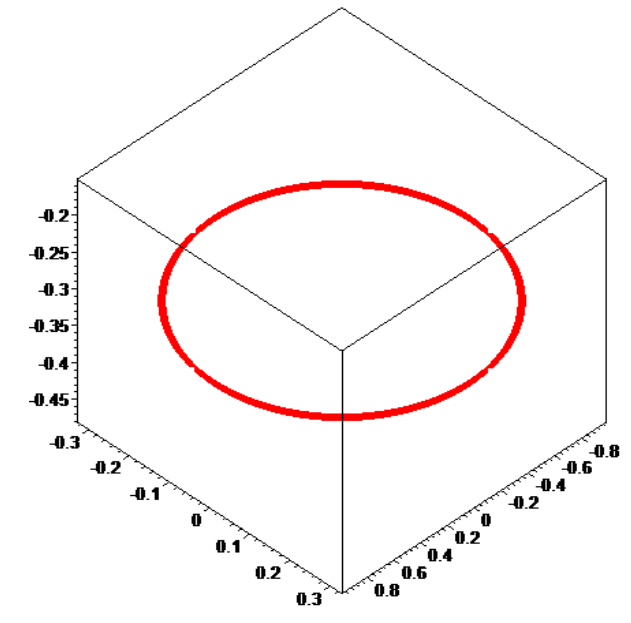

Figure 1. $T^{*} N^{*}$-Smarandache Curve

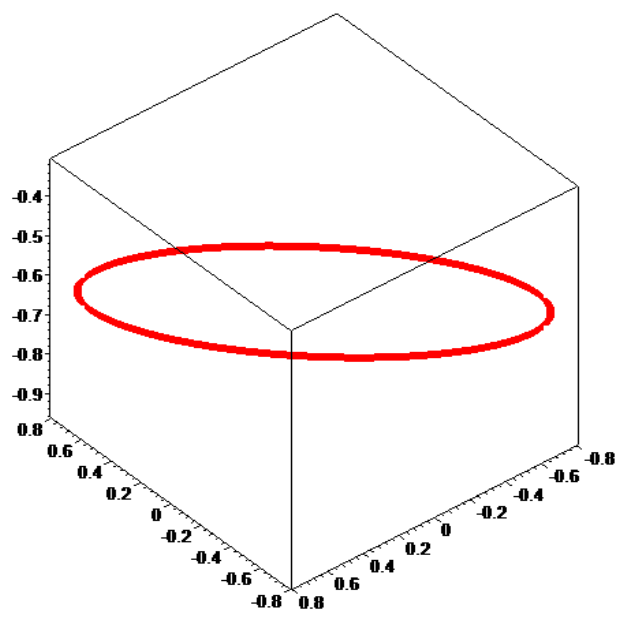

$N^{*} B^{*}$-Smarandache Curve 


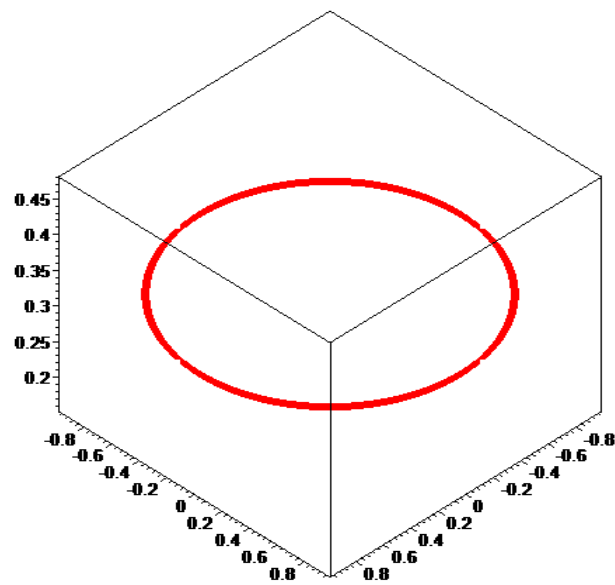

Figure 2. $T^{*} B^{*}$-Smarandache Curve

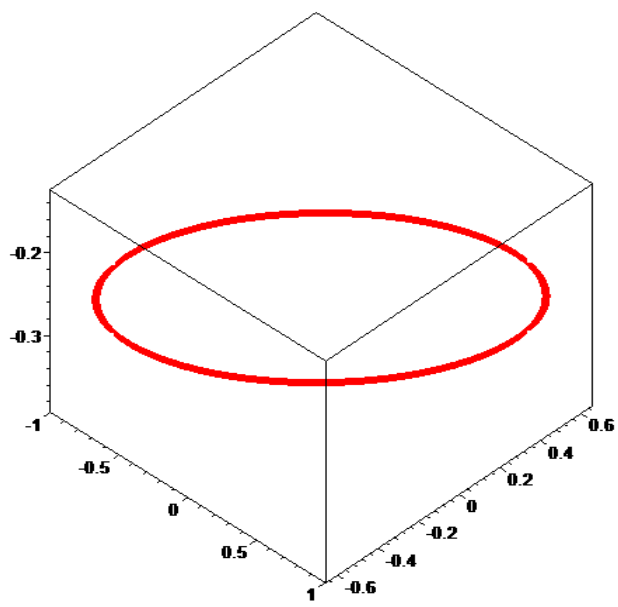

$T^{*} N^{*} B^{*}$-Smarandache Curve

\section{References}

[1] Ali, A. T., Special Smarandache Curves in the Euclidean Space, Intenational Journal of Mathematical Combinatorics, 2(2010), 30-36.

[2] Bektaş, Ö. and Yüce, S., Special Smarandache Curves According to Dardoux Frame in Euclidean 3-Space, Romanian Journal of Mathematics and Computer science, 3(2013), no.1, 48-59.

[3] Bayrak, N., Bektaş, Ö. and Yüce, S., Special Smarandache Curves in $\mathbb{E}_{1}^{3}$, International Conference on Applied Analysis and Algebra, Y1ld1z Techinical University, 209, 2012.

[4] Çalışkan, A. and Şenyurt, S. Smarandache Curves In terms of Sabban Frame of Spherical Indicatrix Curves, Gen. Math. Notes, 31(2015), no.2, 1-15.

[5] Çetin, M., Tuncer, Y. and Karacan M.K., Smarandache Curves According to Bishop Frame in Euclidean 3-Space, Gen. Math. Notes, 20(2014), 50-66.

[6] Hacısalihoğlu, H.H. Differential Geometry, İnönü University, Malatya, Mat. no.7, 1983.

[7] Liu, H. and Wang, F., Mannheim partner curves in 3-space, Journal of Geometry, 88(2008), no.1-2, 120-126(7).

[8] Orbay, K. and Kasap, E., On Mannheim partner curves in, International Journal of Physical Sciences,4 (2009), no.5, 261-264.

[9] Sabuncuoğlu, A. Differential Geometry, Nobel Publications, Ankara, 2006.

[10] Turgut, M. and Yılmaz, S., Smarandache Curves in Minkowski space-time, International Journal of Mathematical Combinatorics, 3(2008), 51-55.

[11] Wang, F., and Liu, H., Mannheim Partner Curves in 3-Space, Proceedings of The Eleventh International Workshop on Diff. Geom., 11(2007), 25-31.

\section{Affiliations}

\section{SÜLEYMAN ŞENYURT}

AdDress: Ordu University,Faculty of Arts and Sciences, Department of Mathematics, Ordu-Turkey. E-MAIL: senyurtsuleyman@hotmail.com

\section{ABDUSSAMET ÇALIŞKAN}

AdDress: Ordu University,Faculty of Arts and Sciences, Department of Mathematics, Ordu-Turkey.

E-MAIL: abdussamet65@gmail.com 